PHYSICAL REVIEW D 92, 115008 (2015)

\title{
LHC constraints on gravitino dark matter
}

\author{
Alexandre Arbey ${ }^{\dagger}$ \\ Centre de Recherche Astrophysique de Lyon, Observatoire de Lyon, \\ Saint-Genis Laval Cedex, F-69561, France; \\ CNRS, UMR 5574, Ecole Normale Supérieure de Lyon, Université de Lyon, \\ Université Lyon 1, F-69622 Villeurbanne Cedex, France; \\ and CERN, CH-1211 Geneva, Switzerland
}

Marco Battaglia*

Santa Cruz Institute of Particle Physics, University of California at Santa Cruz, California 95064, USA and CERN, CH-1211 Geneva, Switzerland

Laura Covi ${ }^{\ddagger}$

Institute for theoretical Physics, Georg-August-Universität Göttingen, D-37077 Göttingen, Germany

Jasper Hasenkamp ${ }^{\S}$

Center for Cosmology and Particle Physics, New York University, New York, New York 10003, USA

\author{
Farvah Mahmoudil \\ Centre de Recherche Astrophysique de Lyon, Observatoire de Lyon, \\ Saint-Genis Laval Cedex, F-69561 France; \\ CNRS, UMR 5574, Université de Lyon, Université Lyon 1, F-69622 Villeurbanne Cedex, France; \\ and CERN, CH-1211 Geneva, Switzerland \\ (Received 11 September 2015; published 11 December 2015)
}

\begin{abstract}
Gravitino dark matter (DM) represents a compelling scenario in supersymmetry (SUSY), which brings together a variety of data from cosmology and collider physics. We discuss the constraints obtained from the LHC on supersymmetric models with gravitino dark matter and the neutralino next-to-lightest SUSY particle, which is the case most difficult to disentangle at colliders from a neutralino lightest SUSY particle forming DM. The phenomenological SUSY model with $19+1$ free parameters is adopted. Results are obtained from broad scans of the phase space of these uncorrelated parameters. The relation between gravitino mass, gluino mass and reheating temperature as well as the derived constraints on these parameters are discussed in detail. This relation offers a unique opportunity to place stringent bounds on the cosmological model, within the gravitino dark matter scenario, from the results of the LHC searches in run2 and the planned high-luminosity upgrade.
\end{abstract}

DOI: 10.1103/PhysRevD.92.115008

PACS numbers: 11.30.Pb, 14.80.Ly, 14.80.Nb, 95.35.+d

\section{INTRODUCTION}

With the discovery of the Higgs boson at the LHC [1,2], collider particle physics moves on to a new phase, where searches for new phenomena will be at the center of the physics program. Dark matter (DM) plays a special role in connecting these searches to cosmology. Not only DM

\footnotetext{
*Corresponding author.

Marco.Battaglia@ucsc.edu

†alexandre.arbey@ens-lyon.fr

taura.covi@theorie.physik.uni-goettingen.de

§asper.hasenkamp@nyu.edu

mahmoudi@in2p3.fr

Published by the American Physical Society under the terms of the Creative Commons Attribution 3.0 License. Further distribution of this work must maintain attribution to the author(s) and the published article's title, journal citation, and DOI.
}

appears to be the most solid indication of new physics obtained so far, but the precision of the determination of its relic density in the Universe, through the study of the cosmic microwave background (CMB) [3], and the increasing sensitivity of the searches for DM scattering in underground experiments [4-6] represent powerful constraints for most models of new physics that offer a DM candidate.

Supersymmetry (SUSY) is one of the most promising and best motivated extensions of the Standard Model of particle physics (SM), providing at the same time a solution to the hierarchy problem within a weakly coupled theory, radiative electroweak symmetry breaking with a light Higgs scalar with properties that, in the decoupling limit, come to resemble those of the Standard Model, and also naturally includes several viable dark matter candidates within its extensive particle spectrum.

Searches conducted at the LHC on the $7+8 \mathrm{TeV}$ run- 1 data have already set some significant constraints on low 
energy SUSY, in particular on the mass of the colored superpartners and on the general mass scale in the case of highly constrained models, such as the constrained MSSM (CMSSM) and the nonuniversal Higgs masses (NUHM) models [7-9]. However, many scenarios with supersymmetric particles within the reach of the LHC runs at 13-14 TeVare totally unprobed at present, in particular when we consider more general SUSY models [10-13] such as the "phenomenological MSSM" (pMSSM), without any assumption on the SUSY breaking mediation mechanism and on unification at high scale. This model gives the most general framework in the minimal SUSY extension of the SM (MSSM) for studying supersymmetric signals and constraints from colliders. It is being adopted by an increasing number of phenomenological [14-27] and experimental [28,29] studies.

The particle most studied in conjunction with SUSY dark matter is the lightest neutralino, $\tilde{\chi}_{1}^{0}$, which provides us with a viable realization of the WIMP mechanism and implies DM signals not only at colliders, but also in direct and indirect detection. However, another very well-motivated SUSY candidate for DM is the gravitino, $\tilde{G}$, the superpartner of the graviton which couples with Planck-suppressed strength to the rest of the model, making it very difficult to detect in direct or indirect DM experiments, in SUSY models with conserved R-parity. Even a small R-parity breaking opens up the possibility of the gravitino decaying DM candidate relaxing part of the cosmological constraints [30]. Colliders offer a unique way to probe the DM sector for scenarios with gravitino dark matter. Therefore, dedicated searches should be actively pursued to fully exploit the LHC reach in these scenarios.

In general, assuming that the gravitino is the lightest SUSY particle (LSP) and forms DM greatly relaxes the DM constraints on the SUSY spectrum, since its abundance is not produced through the WIMP mechanism and does not only depend on the SUSY spectrum, but also on the reheating temperature in the Universe after inflation $[31,32]$. Consequently, the $\tilde{G}$ remains a good candidate for DM even if the spectrum of the superpartners turns out to be much heavier than expected. Nevertheless, we will show that the correlation between the gluino (and in general gaugino) mass and gravitino production ensures that the LHC will be able to probe the gravitino production mechanism for high reheating temperatures above $10^{9} \mathrm{GeV}$ and that the high luminosity LHC program (HL-LHC) may play a crucial role for these tests.

In this paper we present a study of the present and future constraints on SUSY models with gravitino LSP responsible for DM and neutralino next-to-lightest SUSY particle (NLSP). These models are not yet strongly constrained since the current LHC limits on weakly produced SUSY particles are limited in coverage and our scenarios of interest not easy to disentangle from those of neutralino LSP. Indeed, here we consider the parameter space where the neutralino is stable on collider time scales and gives rise to the usual missing transverse energy (MET) signatures at the LHC. After considering the present bounds on the model and how strongly the cosmological and astrophysical constraints limit the parameter space, we assess the capability of the forthcoming LHC runs to test the gravitino production mechanism and to tell the gravitino LSP from the neutralino LSP solutions.

Compared to previous studies, this analysis implements the latest collider constraints, including those from monojet searches which complement the other searches in the regions of the parameter space with degenerate SUSY masses, and we discuss the combination of LHC and other data in the context of the gravitino models. We adopt the pMSSM as a generic MSSM model that, contrary to more constrained models used in the past [33-39] for similar studies, does not imply relations between the masses of the different SUSY particles. This opens up new regions of the parameter space with interesting phenomenology and search opportunities at the LHC which were not available in the (CMSSM), as we demonstrate in this study. The concurrent analysis of the LHC data and the DM direct detection experimental results will be key to identify a neutralino NLSP signal without observation of the decay or to exclude a large fraction of the model parameter space. The combination of the LHC sensitivity on the gluino mass and the requirement of a large enough reheating temperature after inflation for thermal leptogenesis [40-43] is specific to the study performed in the pMSSM, restricts the viable MSSM parameter space and highlights the capability of the LHC to test these cosmological scenarios.

This paper is organized as follows: after discussing the generalities of the gravitino LSP and DM scenario together with the cosmological bounds from nucleosynthesis in Sec. II, we describe our scan strategy for the pMSSM parameter space and the collider and low-energy constraints in Sec. III. Section IV presents the results of our analysis, while in Sec. V we give our conclusions.

\section{GRAVITINO DARK MATTER}

The gravitino is the superpartner of the graviton in models with local supersymmetry [44]. It is the particle most directly related to the effect of SUSY breaking and obtains its mass via the SuperHiggs mechanism [45] as the gauge-fermion of SUSY from any existing source of breaking. The hierarchy between the masses of the superpartners is determined by the particular mediation mechanism, and it can result in a gravitino which is heavier, lighter or even much lighter than the other superpartners. If the gravitino is the lightest SUSY state and therefore absolutely (or sufficiently) stable, it represents a viable candidate for explaining dark matter. The gravitino couplings are set by supergravity and by the MSSM parameters and are inversely proportional to the reduced Planck mass, taken here at its standard value of $M_{P}=2.4 \times 10^{18} \mathrm{GeV}$. The gravitino mass is the only additional parameter needed to describe the gravitino and its interactions. Starting with 
the phenomenological MSSM with 19 parameters set at the electroweak scale, we will add $M_{\tilde{G}}$ as an independent parameter, bringing the total to 20 .

In this paper, we shall focus our analysis on the part of the parameter space where the gravitino is the LSP and a cold DM candidate. We do not consider a very light gravitino, with mass in the $\mathrm{eV}$ to few $\mathrm{keV}$ range, since it is at odds with present cosmological data which strongly disfavor hot or warm dark matter and may only constitute a subdominant DM component [46-49]. In this region of parameters, another (cold) DM candidate is needed and no other supersymmetric particle can fulfill this role. Moreover, a very light gravitino can be a warm thermal relic only in the presence of entropy production with a sufficient dilution factor of the gravitino freeze-out density [50]. Still, this scenario has been widely studied, in particular in relation to the gauge-mediated SUSY breaking mechanism [51,52]. It gives very distinctive signatures at colliders since the NLSP decays promptly leading to photons/jet + MET, which are actively searched by the ATLAS and CMS experiments at the LHC [53-56]. In the scenarios we explore here, the next-to-lightest SUSY particle (NLSP) often mimicks a stable LSP, even if the NLSP can be in principle charged under the SM group and therefore cannot be stable on cosmological scales. We assume the NLSP to be the lightest neutralino, $\tilde{\chi}_{1}^{0}$, which is the case most difficult to disentangle at colliders from the scenario of neutralino LSP and dark matter particle.

Although very weakly coupled, gravitinos can be produced in substantial numbers in the primordial thermal bath from scattering of SUSY particles, in particular the colored or electroweakly charged states. Usually the interactions involving gluinos or gauginos gives the dominant contribution since they are some of the most abundant and strongly coupled particles and interact with gravitinos by an effective dimension- 5 operator, leading to a yield proportional to the highest equilibrium temperature of the thermal bath [31,32]. For a light gravitino, this contribution is dominated by the Goldstino component of the gravitino and given by

$$
\begin{aligned}
\Omega_{\tilde{G}}^{\text {th }} h^{2}= & 0.83 \frac{T_{\mathrm{RH}}}{10^{9} \mathrm{GeV}}\left(\frac{m_{3 / 2}}{1 \mathrm{GeV}}\right)^{-1} \sum_{i=1}^{3} \gamma_{i}\left(T_{\mathrm{RH}}\right) \\
& \times\left(\frac{M_{i}}{300 \mathrm{GeV}}\right)^{2},
\end{aligned}
$$

where $\gamma_{i}\left(T_{\mathrm{RH}}\right)$ are numerical factors including the dependence on the gauge coupling for each SM gauge group and the evolution of the gauge couplings and gaugino masses $M_{i}$ to the scale of the reheating temperature $T_{\mathrm{RH}}$. To do so, we used here only one-loop renormalization group equations (RGEs) to capture the main effect and we define, following [32],

$$
\gamma_{i}(T)=y_{i} \frac{g_{i}^{6}(T)}{g_{i}^{4}\left(M_{i}\right)} \log \left[\frac{k_{i}}{g_{i}(T)}\right]
$$

with $\left(y_{i}, k_{i}\right)$ equal to $(4.276,1.271), \quad(1.604,1.312)$, $(0.653,1.266)$ for the SM gauge groups $S U(3), S U(2)$ and $U(1)$, respectively. It is clear from these factors that the gluinos often give the largest contribution, due to their multiplicity and their larger gauge coupling. The expression given in Eq. (1) is valid only in the perturbative regime, and becomes less reliable at low reheat temperatures, where $g_{3}$ becomes large. Note though, that for low $T_{\mathrm{RH}}$, in the region of supersymmetric masses we will explore, gaugino scatterings do not provide the dominant contribution to the gravitino relic density and therefore an order 1 determination of this part is sufficient for our purposes.

Processes involving scalar SUSY particles, like squarks and sleptons, include also dimension-4 type couplings, which generate a yield independent of the thermal bath temperature, but those contributions are often negligible, unless a strong hierarchy between scalar and gaugino masses is assumed [57,58]. The contribution from the NLSP decay after freeze-out can be quite substantial, especially for the case of a bino-like neutralino NLSP. Such a contribution is given by [59-61]

$$
\Omega_{\tilde{G}}^{\mathrm{SW}} h^{2}=\left(\frac{m_{3 / 2}}{M_{\chi}}\right) \Omega_{\chi} h^{2},
$$

where $M_{\chi}$ is the mass of the lightest neutralino and $\Omega_{\chi} h^{2}$ its present energy density, if the neutralino would be stable, set by the freeze-out process.

In determining the relic gravitino density we consider the thermal contribution from gaugino scattering given in Eq. (1) as well as that arising from neutralino decay out of equilibrium in Eq. (3). For any value of the pMSSM parameters and gravitino mass, a specific value of the reheat temperature allows us to match the observed DM abundance, as long as the contribution from NLSP decay is smaller than the observed DM density. In the pMSSM parameter region where the neutralino relic density exceeds the DM energy density, an upper bound on the gravitino mass also appears. This bound may be stronger than our request that the gravitino is the LSP. In general, there is a maximal value of the reheating temperature allowed at any parameter point, and possibly also a maximal gravitino mass, in order to avoid gravitino density overclosure.

The total gravitino density can be written in a simplified form as

$$
\Omega_{\tilde{G}} h^{2}=A \frac{T_{\mathrm{RH}}}{m_{3 / 2}}+B m_{3 / 2},
$$

where the parameters $A$ and $B$ are functions of the pMSSM parameters, as can be read from Eqs. (1) and (3). $A$ depends mostly on the gaugino masses $M_{1}, M_{2}$ and $M_{3}$, while $B$ contains the neutralino relic density and depends directly on the neutralino composition. Requiring these two 
mechanisms to give rise to the full dark matter energy density can be recast in a simple quadratic equation for $m_{3 / 2}$ :

$$
B m_{3 / 2}^{2}-\Omega_{\mathrm{CDM}} h^{2} m_{3 / 2}+A T_{\mathrm{RH}}=0
$$

which has two real roots for $m_{3 / 2}$ only when

$$
T_{\mathrm{RH}} \leq \frac{\left(\Omega_{\mathrm{CDM}} h^{2}\right)^{2}}{4 A B}
$$

and this value provides the maximal possible reheating temperature for any gravitino mass. Note that the rhs of Eq. (6) depends only on the pMSSM parameters through the $A$ and $B$ coefficients. The maximal reheating temperature is realized when the CDM density is provided equally by the two production mechanisms and the gravitino mass is fixed to the value

$$
m_{3 / 2}=\frac{\Omega_{\mathrm{CDM}} h^{2}}{2 B}=\frac{\Omega_{\mathrm{CDM}} h^{2}}{\Omega_{\chi} h^{2}} \frac{M_{\chi}}{2} .
$$

If $\Omega_{\chi} h^{2}<\Omega_{\mathrm{CDM}} h^{2} / 2$, this particular value of $m_{3 / 2}$ is excluded by the requirement $m_{3 / 2}<M_{\chi}$. For values of the gravitino mass lower than in Eq. (7), the bound on $T_{\mathrm{RH}}$ gets stronger. Other cosmological constraints restrict the region of large $m_{3 / 2}$ and in general in any point of the pMSSM parameter space set a stronger bound on $T_{\mathrm{RH}}$ compared to that given by Eq. (6). This constraint is given by

$$
T_{\mathrm{RH}} \leq \frac{m_{3 / 2}}{A}\left(\Omega_{\mathrm{CDM}} h^{2}-B m_{3 / 2}\right),
$$

and it depends on both the gravitino mass and the pMSSM parameters.

The reheating temperature of the Universe is an important parameter in the cosmological evolution and depends on both the particular inflationary model and the reheating process after inflation. It strongly influences the possible mechanisms of baryogenesis, needed to generate the present baryon asymmetry starting from a symmetric initial state. In particular, the thermal leptogenesis mechanism [62] relies on the presence of heavy right-handed Majorana neutrinos in the thermal bath. These decay out-of-equilibrium giving rise to a nonvanishing lepton and then a baryon asymmetry.

Thermal leptogenesis can produce the observed baryon number only if the reheating temperature is sufficiently high, above approximately $2 \times 10^{9} \mathrm{GeV}$ [40,42]. With a mild tuning in the seesaw formula and exploiting flavor effects, this bound can be relaxed by an order of magnitude $[43,63]$ and even more so in the case of resonant $C P$ violation, due to nearly degenerate $\mathrm{RH}$ neutrino masses [64,65]. While these bounds are not intrinsic to the gravitino DM hypothesis, they are a desirable addition to big bang cosmology. We will discuss how the requirement of high $T_{\mathrm{RH}}$ can be used in these specific scenarios to place strong constraints on the SUSY spectrum, when it is compared to the reheating temperature needed to generate the correct gravitino DM abundance. Indeed, as can be inferred from Eq. (1), light degenerate gauginos reduce gravitino production and allow for the highest possible $T_{\mathrm{RH}}$ around $10^{9} \mathrm{GeV}[66,67]$. This makes possible to interpret collider data as tests of the simplest thermal leptogenesis hypothesis.

\section{A. Cosmological constraints}

The requirement of a consistent cosmology sets important constraints on models with a very weakly interacting particle like the gravitino. Indeed, even if the gravitino is not the LSP and therefore unstable, it may decay very late in the cosmological history leading to the disruption of big bang nucleosynthesis (BBN), the so-called "gravitino problem" [68-70]. It is interesting to point out that these scenarios may be seen as an asset, since these late decays might solve another outstanding problem, that of the amount of cosmic lithium formed after the big bang [71]. In our scenario the gravitino is the LSP and so the long-lived particle becomes the neutralino NLSP. Due to the Planck scale suppression, the NLSP lifetime can easily exceed $1 \mathrm{~s}$ and the decay of the out-of-equilibrium NLSPs again takes place during BBN, thus affecting the abundance of light elements [72]. For a neutral long-lived particle like the neutralino, the main processes affecting BBN are photo or hadrodissociation, which depend on the number density of the decaying particle and its branching fraction into photon or hadrons. For short lifetimes the constraints from hadrodissociation are stronger than those given by photodissociation, which takes over at $\tau>10^{4}-10^{6} \mathrm{~s}[73,74]$. The constraints for neutral relic with different values of the hadronic branching ratio and decaying particle mass were computed by running a BBN code including the neutral particle decay channel into quark and antiquark pairs in [74]. Here, we use those generic constraints and translate them to the special case of the neutralino, similarly to what was done in $[19,75]$. In order to do that we need to compute the decaying branching fractions and the freeze-out density of the NLSP for every point of the pMSSM parameter space.

The decay rates of a general neutralino NLSP into a gravitino LSP were calculated analytically including all possible three-body decays in [75]. The neutralino decays dominantly into a gravitino and a gauge boson. The hadronic channel into gravitino with a quark and antiquark pair arises most of the time from an intermediate gauge or Higgs boson, as long as the scalar quarks are not too light. So the most important hadronic channel for the neutralino consists of a three-body decay, where part of the energy is carried away by the inert gravitino, instead of the simple two-body decay into quark and antiquark, which was assumed in [74] for the neutral relic. Therefore the 
quark-pair momentum distribution in neutralino decay is softer than in a two-body decay and we therefore regard the limits given in [74] as possibly too strong for our scenario. Indeed, if the intermediate gauge boson is on shell before decaying into a quark-antiquark pair, the number of hadronic particles produced is expected to be independent of the gauge boson energy or momentum. Moreover, if the hadrons produced thermalize before interacting with light elements, any dependence on the initial spectrum or the mass of the mother particle is washed out. In this spirit, the work of Ref. [19] uses the bounds of Jedamzik for a decaying particle mass of $100 \mathrm{GeV}$, disregarding the dependence on this mass and correcting the electromagnetic bounds by subtracting the energy carried away by the gravitino. In this study we implement both sets of constraints on our scans: first the limits given by [74] as a function of the hadronic branching ratio and decaying particle mass, and then the slightly weaker limits of Ref. [19]. Indeed, since in this analysis we include also regions of the parameter space where the $Z$ or Higgs boson cannot be on shell, i.e. the region where $M_{1}-m_{3 / 2}<M_{Z}$, $M_{h}$, we consider the latter bounds as too conservative in some part of the parameter space. The comparison between the two sets of bounds, with one appearing to be too strong and the other too weak in part of the parameter space, provides us with an estimation on the sensitivity of the results on the precise implementation of these constraints.

In general, BBN bounds exclude regions where the density of the decaying particle as a function of its lifetime is too large. Indeed in the early stages of BBN, corresponding to lifetimes below $10^{2} \mathrm{~s}$, the constraints are very weak, or even nonexistent, while after the production of light elements, like deuterium, helium or lithium, the effect of hadrodissociation becomes stronger. The lifetime of the NLSP in our scenario is always related to the gravitino mass, since for a light gravitino its couplings are proportional to $1 / m_{3 / 2}$. These bounds can be translated in general into an upper bound on the gravitino mass, as a function of the NLSP density at freeze-out. For a bino-like neutralino NLSP lifetime, above the $Z$ threshold, we have

$$
\begin{aligned}
\Gamma_{\tilde{\chi}_{1}^{0} \rightarrow \gamma / Z \psi_{3 / 2}} & =\frac{1}{48 \pi M_{P}^{2}} \frac{M_{\chi}^{5}}{m_{3 / 2}^{2}} \\
& =(57 \mathrm{~s})^{-1}\left(\frac{M_{\chi}}{1 \mathrm{TeV}}\right)^{5}\left(\frac{m_{3 / 2}}{10 \mathrm{GeV}}\right)^{-2} .
\end{aligned}
$$

For gravitino masses larger than about $1 \mathrm{GeV}$, the NLSP typically decays during BBN and affects its predictions. It is possible to shorten the NLSP lifetime by varying the value of the gravitino mass, independently of the decaying neutralino mass or composition.

We use the results of [75] to implement the hadronic constraints on the model, deriving the branching fraction for the neutralino decays into a gravitino and quark-antiquark pair for each point of the pMSSM parameter space scan. This branching fraction is shown as a function of the neutralino mass for our pMSSM scan points in Fig. 1. The nature of the neutralino is highlighted, with the bino-, wino- and Higgsino-like components defined as the points with the corresponding entry squared in the neutralino mixing matrix exceeding 0.75 . The minimal hadronic branching fraction for fixed neutralino mass is obtained for the neutralino composition of a pure photino, since in this case the contributions through intermediate $Z$ and Higgses are absent.

We obtain the neutralino branching fraction into hadrons as follows. First, we estimate the neutralino lifetime considering the decay into the gravitino and a photon, which is always open, keeping all kinematical factors in this channel. Then, we compute all the other decay widths, e.g. into $Z$ and $h$, by setting the gravitino mass to zero in the kinematical factors. Since the photon or another open decay channel dominates, when thresholds are encountered, this approximation holds below the per-mille level in all points and gives a rather conservative estimate of the hadronic branching fraction. Next, we compute the decay rate into the hadronic channel taking into account the neutralino composition and the presence of off-shell or on-shell intermediate bosons. If the intermediate particles are on shell, the result is well approximated by the decay rate into that particle times the corresponding branching fraction. But our results extend also into the regions with off-shell intermediate states. In this case, we integrate the branching fraction for energies of the quark-antiquark pairs above their mass threshold. As discussed in [75] this gives, below the $Z$ threshold, a result 3 times larger than taking a threshold of $2 \mathrm{GeV}$, corresponding to the production in the

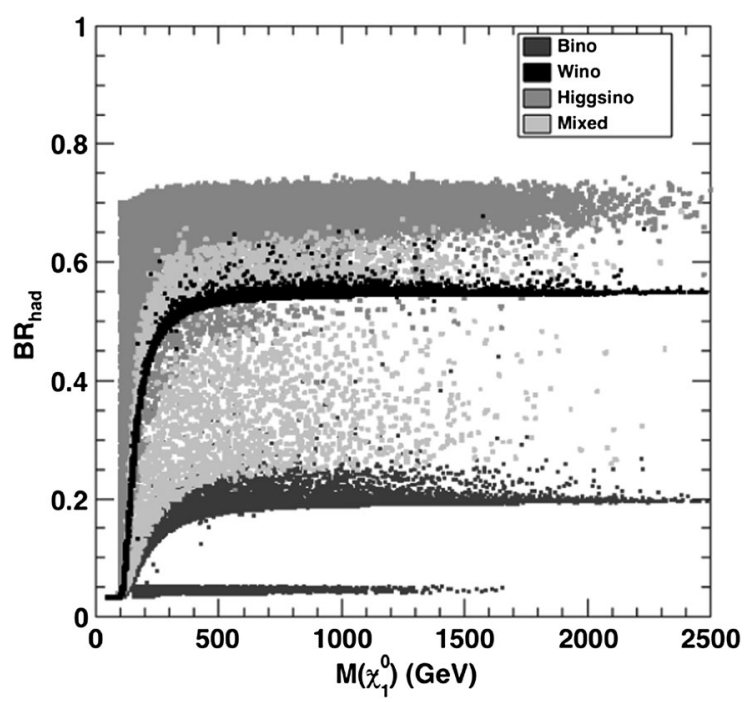

FIG. 1. Neutralino hadronic decay branching fraction as a function of the neutralino mass for the accepted pMSSM scan points, with the nature of the neutralino having the largest contribution in each region highlighted. 
decay of hadrons instead of mesons. At leading order the branching fraction is independent of the gravitino mass, since all rates are proportional to the same factor $1 / \mathrm{m}_{3 / 2}^{2}$. Only for large gravitino masses near thresholds does this parameter play an important role also in the phase-space factors.

Photodissociation constraints are very similar in most of the parameter space and mostly independent on the specific values of the purely electromagnetic branching ratios, since the decay into the gravitino and a photon has always a branching fraction larger than $30 \%$ and the hadronic bounds in Ref. [74] include the photodissociation effects from charged hadrons.
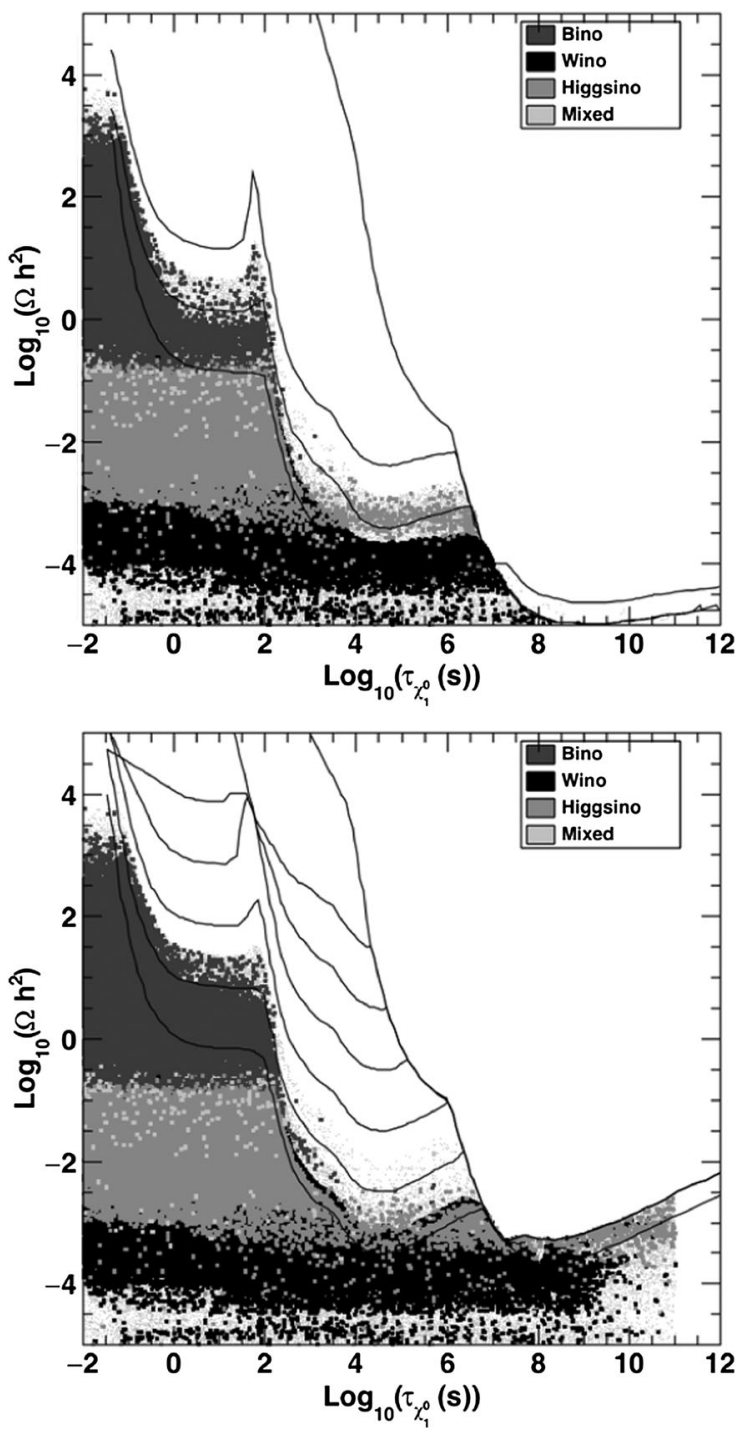

FIG. 2. Neutralino relic density as a function of the neutralino lifetime for the accepted pMSSM scan points, with the nature of the neutralino having the largest contribution in each region highlighted. The BBN constraints obtained following Ref. [74] (top) and Ref. [19] (bottom) are shown by the continuous lines corresponding to limits for neutralino hadronic branching fractions of $100 \%, 10 \%, 1 \%$ and $0 \%$ from bottom to top.
The calculation of the cosmological bounds require one to implement the number density of the neutralino NLSP before decay. This is evaluated by considering the freezeout process of the neutralino and solving the corresponding Boltzmann equation using the SUPERISO RELIC code [76]. The neutralino NLSP density is determined by the 19 pMSSM parameters and it is independent of the gravitino mass. Therefore we can find iteratively the largest NLSP lifetime and thus the largest gravitino mass compatible with BBN and the NLSP number density for a set of pMSSM parameters, as discussed in the next section. The maximal allowed value for the gravitino mass at any chosen point in the pMSSM parameter space is usually smaller than the general upper bound given in Eq. (7) and therefore we can apply Eq. (8) to obtain a tighter bound on the reheating temperature $T_{\mathrm{RH}}$. The distribution of the neutralino relic
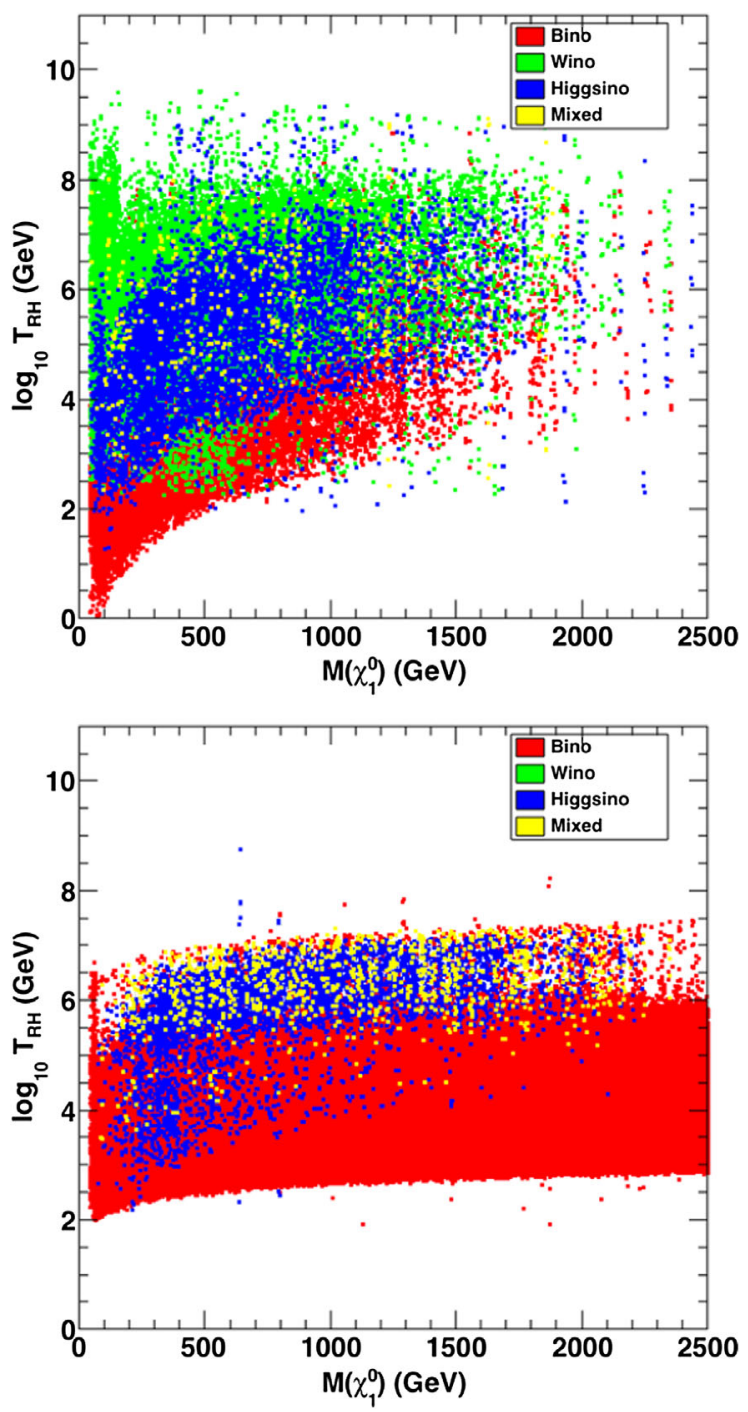

FIG. 3 (color online). Reheating temperature as a function of the NLSP neutralino mass for the accepted pMSSM (top) and cMSSM (bottom) scan points, with the nature of the neutralino having the largest contribution in each region highlighted. 
density as a function of its lifetime for the pMSSM scan points is given is Fig. 2. The dependence of the relic density of the neutralino from its composition clearly appears: a bino neutralino has naturally a larger abundance and therefore as an NLSP must have a shorter lifetime than a wino and Higgsino neutralino in order to not disrupt BBN. We expect that wino/Higgsino-like NLSPs can allow for a larger maximal gravitino mass, and so also a larger reheating temperature than a bino NLSP. This can be verified on our scans by studying the distribution of the reheating temperature as a function of the NLSP mass and selecting the NLSP nature. Figure 3 shows the results obtained contrasting the case of the pMSSM scan to those of the cMSSM. In the pMSSM parameter space, points with wino- and Higgsino-like NLSP accommodate values of $T_{\mathrm{RH}}$ in the range $10^{6}-5 \times 10^{9} \mathrm{GeV}$. On the contrary, the bino-like population of the cMSSM points does not offer solutions with $T_{\mathrm{RH}}$ values in excess of $10^{7} \mathrm{GeV}$.

For neutralino lifetimes longer than $10^{10} \mathrm{~s}$, constraints from $\mathrm{CMB}$ distortion also play a role and start to be more stringent than the BBN constraints, see e.g. [77]. Since we can obtain such extremely large lifetime values only for very few fine-tuned points of our parameter scan, as in the degenerate gravitino-NLSP scenario [78], we implement an upper cut at $\tau=10^{11} \mathrm{~s}$.

\section{ANALYSIS}

\section{A. MSSM scans}

The phenomenological MSSM (pMSSM) is the most general MSSM scenario with R-parity, $C P$ conservation and minimal flavor violation [10] and its properties are defined by 19 independent parameters. This analysis is based on a flat scan where these parameters are varied within the ranges given in Table I. The gravitino mass is added as an additional parameter. The ranges of the SUSY particle masses have been made broader compared to previous studies $[16,18,19]$ to achieve a better assessment of the capability of the LHC at $13-14 \mathrm{TeV}$, which will probe the masses of strongly interacting SUSY particles up to $2-3 \mathrm{TeV}$. For this analysis a total of $7 \times 10^{7} \mathrm{pMSSM}$ points have been generated and analyzed.

In our analysis, the SUSY mass spectra and couplings are generated from the input pMSSM parameters with SOFTSUSY 3.3.3 [79]. Only points having the lightest neutralino, $\tilde{\chi}_{1}^{0}$, to be the next lightest SUSY particle to the gravitino and the lightest Higgs mass in the range $122-128 \mathrm{GeV}$ are accepted. Particle decay widths are calculated using HDECAY 5 [80] and SDECAY [81].

In order to contrast the gravitino phenomenology in the parameter space of the pMSSM to that of constrained MSSM models, we also perform scans of the constrained MSSM (CMSSM) [82,83] where we vary the $M_{0}, M_{1 / 2}$, $\tan \beta$ and $A_{0}$ parameters in the ranges indicated in Table II.
TABLE I. Range of the pMSSM parameters adopted in the scans (in $\mathrm{GeV}$ when applicable).

\begin{tabular}{lc}
\hline \hline Parameter & Range \\
\hline $\tan \beta$ & {$[1,60]$} \\
$M_{A}$ & {$[50,5000]$} \\
$M_{1}$ & {$[-5000,5000]$} \\
$M_{2}$ & {$[-5000,5000]$} \\
$M_{3}$ & {$[0,5000]$} \\
$A_{d}=A_{s}=A_{b}$ & {$[-15000,15000]$} \\
$A_{u}=A_{c}=A_{t}$ & {$[-15000,15000]$} \\
$A_{e}=A_{\mu}=A_{\tau}$ & {$[-15000,15000]$} \\
$\mu$ & {$[-5000,5000]$} \\
$M_{\tilde{e}_{L}}=M_{\tilde{\mu}_{L}}$ & {$[0,5000]$} \\
$M_{\tilde{e}_{R}}=M_{\tilde{\mu}_{R}}$ & {$[0,5000]$} \\
$M_{\tilde{\tau}_{L}}$ & {$[0,5000]$} \\
$M_{\tilde{\tau}_{R}}$ & {$[0,5000]$} \\
$M_{\tilde{q}_{1 L}}=M_{\tilde{q}_{2 L}}$ & {$[0,5000]$} \\
$M_{\tilde{q}_{3 L}}$ & {$[0,5000]$} \\
$M_{\tilde{u}_{R}}=M_{\tilde{c}_{R}}$ & {$[0,5000]$} \\
$M_{\tilde{t}_{R}}$ & {$[0,5000]$} \\
$M_{\tilde{d}_{R}}=M_{\tilde{s}_{R}}$ & {$[0,5000]$} \\
$M_{\tilde{b}_{R}}$ & {$[0,5000]$} \\
\hline \hline
\end{tabular}

For each accepted point, we compute the flavor observables and the muon anomalous magnetic moment with SUPERIso v3.3 [84,85], and the neutralino relic density with SuPERIso ReLIC [76], where the gravitino related calculations have also been implemented. To allow for comparisons between the gravitino LSP and the neutralino LSP scenarios, we compute the neutralino direct detection scattering cross sections using the MicrOMEGAs code [86].

The constraints from the LHC SUSY searches at $7+8$ and their projection to $14 \mathrm{TeV}$ are obtained through the analysis of inclusive SUSY events generated by PYTHIA 8.150 [87] with the CTEQ6L1 parton distribution functions [88]. SUSY particle production accompanied by a hard jet, relevant to monojet searches, is simulated using MADGRAPH 5 [89] followed by PYTHIA for hadronization, as described in [24]. The physics objects of the signal events are obtained with a parametric simulation of the LHC detector response performed with DELPHES 3.0 [90].

For each pMSSM point, we infer the maximal gravitino mass consistent with the BBN constraints and leading to a

TABLE II. Range of the cMSSM parameters adopted in the scans (in $\mathrm{GeV}$ when applicable). Both signs of $\mu$ have been considered.

\begin{tabular}{lc}
\hline \hline Parameter & Range \\
\hline $\tan \beta$ & {$[2,68]$} \\
$M_{0}$ & {$[0,10000]$} \\
$M_{1 / 2}$ & {$[0,10000]$} \\
$A_{0}$ & {$[-10000,10000]$} \\
\hline \hline
\end{tabular}


TABLE III. Summary of the constraints from flavor physics and muon anomalous magnetic moment.

\begin{tabular}{lc}
\hline \hline & $2.63 \times 10^{-4}<\mathrm{BR}\left(B \rightarrow X_{s} \gamma\right)<4.23 \times 10^{-4}[92]$ \\
& $1.3 \times 10^{-9}<\mathrm{BR}\left(B_{s} \rightarrow \mu^{+} \mu^{-}\right)_{\text {untag }}<4.5 \times 10^{-9}[93-95]$ \\
Flavor & $0.33 \times 10^{-4}<\mathrm{BR}\left(B_{u} \rightarrow \tau \nu\right)<1.95 \times 10^{-4}[96,97]$ \\
& $4.7 \times 10^{-2}<\mathrm{BR}\left(D_{s} \rightarrow \tau \nu\right)<6.1 \times 10^{-2}[92,98]$ \\
$(g-2)_{\mu}$ & $0.985<R_{\mu 23}<1.013[99]$ \\
\hline \hline
\end{tabular}

relic density $\Omega_{\tilde{G}} h^{2} \sim 0.11$, in agreement with the Planck CMB data [3]. First, we derive the maximal neutralino lifetime using the BBN limits given in Sec. II A from the computed neutralino relic density and hadronic branching ratio. The corresponding gravitino mass is then obtained through an iterative procedure from the maximal NLSP lifetime. Once the maximal gravitino mass is obtained, we randomly scan over the gravitino mass values within a range comprised between $1 / 1000$ and 10 times the maximal gravitino mass and keep ten of such gravitino mass values for each pMSSM point. If the maximal gravitino mass cannot be obtained, we vary randomly the gravitino mass logarithmically between $0.1 \mathrm{MeV}$ and the neutralino mass. We then compute the gravitino relic density from NLSP decay as given in Eq. (3) and check if it is smaller than or equal to the DM relic density. Since the neutralino relic density is diluted by the ratio of the gravitino to the neutralino mass and the BBN bounds are stronger for larger NLSP density, this is always the case. To retrieve a relic density compatible with the cosmological observations, we compute by inverting Eq. (1) the reheating temperature, $T_{\mathrm{RH}}$, required to increase the gravitino abundance up to the value corresponding to the Planck observations.

In addition, we include a set of points for which the lightest neutralino is a photino, i.e. a mixture of bino and wino with the dominant decay mode to a gravitino and a photon. These states can be obtained at tree level for $M_{1}=$ $M_{2}$ and $\left|M_{1}\right| \ll|\mu|$. However, the neutralino mass matrix receives higher order corrections, which modify this condition. Therefore, we use a specific scan where we first impose $M_{1}=M_{2}$, and then iteratively adjust the value of $M_{2}$ to retrieve the pure photino solution.

\section{B. Collider and low-energy constraints}

The analysis of the viable MSSM parameter space requires the implementation of the available constraints from different sectors.

First the constraints from low energy observables are imposed to the generated points. Table III summarizes the most recent values for the low energy $95 \%$ C.L. limits, obtained by including experimental and theoretical uncertainties. In particular, the branching fraction of the decay $B_{s} \rightarrow \mu^{+} \mu^{-}$is sensitive to scalar and pseudoscalar operators, and provides us with a bound on the $\tan \beta$ and $M_{A}$ parameters [91]. The value of the inclusive $\operatorname{BR}\left(B \rightarrow X_{s} \gamma\right)$ is also sensitive to charged Higgs and chargino/stop contributions at loop level, thus constraining $\tan \beta, M_{A}$, as well as the stop and chargino masses. These constraints remove $\simeq 12 \%$ of the accepted pMSSM points in our scans.

With $\simeq 25 \mathrm{fb}^{-1}$ of statistics collected by both the ATLAS and CMS experiments at $7+8 \mathrm{TeV}$ during the LHC run-1, a vast array of searches for the production and decay of new particles has been performed. In particular, the analysis of channels with missing transverse energy (MET), including jets + MET, leptons + MET and monojets, have excluded a sizable fraction of the MSSM parameter space. With the start of run-2 at $13 \mathrm{TeV}$ and the perspectives of increased luminosity, the LHC will put SUSY scenarios through a crucial, although possibly not yet definitive, test. In this study, we consider the bounds obtained from the analyses summarized in Table IV. Although these are not exhaustive of the signal topologies investigated by the ATLAS and CMS searches, they cover the SUSY signatures with the highest sensitivity and are largely uncorrelated. For each accepted pMSSM point, we simulate an inclusive SUSY event sample and perform a parametric simulation for the event reconstruction, as discussed above. Signal selection cuts corresponding to each of the analyses are applied to these simulated signal events. The number of SM background events in the signal regions are taken from the estimates reported by the experiments. The $95 \%$ confidence level (C.L.) exclusion in the presence of background only is determined using the C.L. method [101].

These results are projected to $14 \mathrm{TeV}$ for 300 and $3000 \mathrm{fb}^{-1}$ of integrated luminosity, by generating events

TABLE IV. Summary of the analyses used to assess the observability of the pMSSM points by the LHC SUSY searches.

\begin{tabular}{lccc}
\hline \hline Channel & Experiment & Sensitivity & Ref. \\
\hline jets + MET & ATLAS & $\tilde{g}, \tilde{q}$ & {$[102]$} \\
$2 \ell+$ jets + MET & ATLAS & $\tilde{t}, \tilde{q}$ & {$[103]$} \\
$1 \ell+$ b-jet(s) + MET & ATLAS & $\tilde{t}$ & {$[104]$} \\
b-jets + MET & ATLAS & $\tilde{t}, \tilde{b}$ & {$[105]$} \\
$2 \ell+$ MET & ATLAS & $\tilde{\chi}^{0} \tilde{\chi}^{ \pm}$ & {$[106]$} \\
$3 \ell+$ MET & ATLAS & $\tilde{\chi}^{0} \tilde{\chi}^{ \pm}$ & {$[107]$} \\
$1 \ell+b b+$ MET & ATLAS & $\tilde{\chi}^{0} \tilde{\chi}^{ \pm}$ & {$[108]$} \\
Monojet + MET & ATLAS & $\tilde{\chi} \tilde{\chi}, \tilde{q} \tilde{q}$ & {$[109]$} \\
Monojet + MET & CMS & $\tilde{\chi} \tilde{\chi}, \tilde{q} \tilde{q}$ & {$[110]$} \\
$H / A \rightarrow \tau \tau$ & CMS & $H, A$ & {$[111]$} \\
\hline \hline
\end{tabular}


at $14 \mathrm{TeV}$ and rescaling the $8 \mathrm{TeV}$ backgrounds by the corresponding increase in cross section and signal cut acceptance at the higher energy. The constraints derived from these searches exclude a significant fraction of the gravitino pMSSM points, from $22 \%$ at $8 \mathrm{TeV}$ to $75 \%$ and $85 \%$ at $14 \mathrm{TeV}$ with 300 and $3000 \mathrm{fb}^{-1}$, respectively. The gluino mass is of particular importance in relation to the gravitino relic density and the reheating temperature. Figure 4 shows the distribution of the gluino mass for the points not excluded by the searches for the different energies and data sets considered here. The gluino mass values at which more than $95 \%$ of the scan points below that mass are rejected by the LHC searches, or will be in case of a negative result, is $M_{\tilde{g}}=840,1900$ and $2300 \mathrm{GeV}$ for $25 \mathrm{fb}^{-1}$ at $8 \mathrm{TeV}, 300 \mathrm{fb}^{-1}$ and $3000 \mathrm{fb}^{-1}$ at $14 \mathrm{TeV}$, respectively. We notice here that, for the general supersymmetric spectra realized in the pMSSM, values of the gluino mass below $1 \mathrm{TeV}$ are still viable, and that the next runs will be able to extend the sensitivity beyond $2 \mathrm{TeV}$.

Since the gravitino LSP implies different constraints from dark matter, it is interesting to contrast the parameter space allowed in this scenario to that of the MSSM with neutralino LSP, after the LHC searches. The main difference between the two models arises from the dark matter relic density constraint, which severely restricts the MSSM parameter space with neutralino LSP and modifies the occurrence of neutralinos of different nature and the relations of the masses of the other SUSY particles to that of the lightest neutralino. In the neutralino LSP scenario, bino-like neutralinos are relatively disfavored by loose relic density constraint, because in general they lead to too large relic density. But imposing the tight relic density constraint

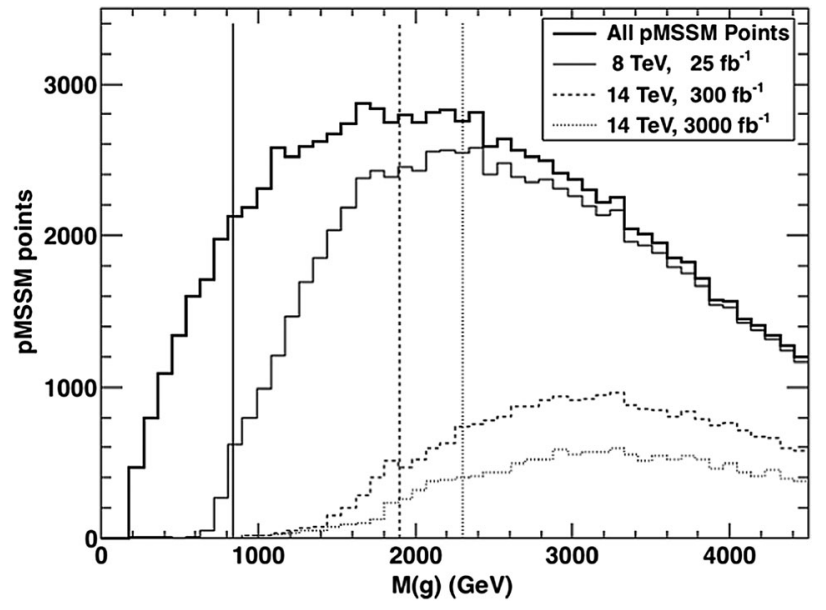

FIG. 4. Distribution of the gluino mass from the pMSSM scans with LSP gravitino and NLSP neutralino showing all the accepted points (black line) and those not excluded with $25 \mathrm{fb}^{-1}$ of $7+$ $8 \mathrm{TeV}$ data (grey line), $300 \mathrm{fb}^{-1}$ (dashed line) and $3000 \mathrm{fb}^{-1}$ of $14 \mathrm{TeV}$ data (dotted line). The vertical lines indicate the masses at which more than $95 \%$ of our scan points with gluino mass below those values are, or will be, excluded by the LHC data. highlights the bino-like scenario, while the wino- and Higgsino-like scenarios become less attractive at the LHC since in these two cases the correct $\Omega_{\chi} h^{2}$ is naturally reached only for neutralino masses well beyond $1 \mathrm{TeV}$, thus pushing the SUSY spectrum to high masses. In the wino scenario more complex decay chains of strongly interacting SUSY particles occur also more frequently. In the bino scenario, it is necessary to have coannihilations, which means SUSY particles with masses close to that of the neutralino LSP are required. This has important consequences for the SUSY detectability at LHC, since compressed spectra or SUSY particles at high mass scales become favored by the tight relic density bound in the neutralino DM scenario.

Here, we use two sets of $\Omega_{\gamma} h^{2}$ constraints for the pMSSM points with neutralino LSP. First, we apply a tight bound requiring the two values to be in agreement allowing for systematic uncertainties, i.e. $0.090<\Omega_{\chi} h^{2}<0.163$, and assuming that the LSP neutralino saturates the observed dark matter. We also consider a looser bound by simply requesting that the neutralino relic density does not exceed the upper bound on the dark matter density from the PLANCK CMB, i.e. $10^{-5}<\Omega_{\chi} h^{2}<0.163$, again after accounting for systematic uncertainties. This allows for other sources of dark matter in addition to the neutralino. Of the pMSSM points we have studied fulfilling the flavor physics and lowenergy data constraints, $1.7 \%$ and $57 \%$ satisfy the tight and loose constraints, respectively. It is therefore clear that some amount of tuning is needed to obtain the right neutralino dark matter density. Moreover, the tight $\Omega_{\chi} h^{2}$ constraint gives only $6.5 \%$ of wino neutralinos, with $56 \%$ Higgsinos and $26 \%$ bino. The loose constraint selects a
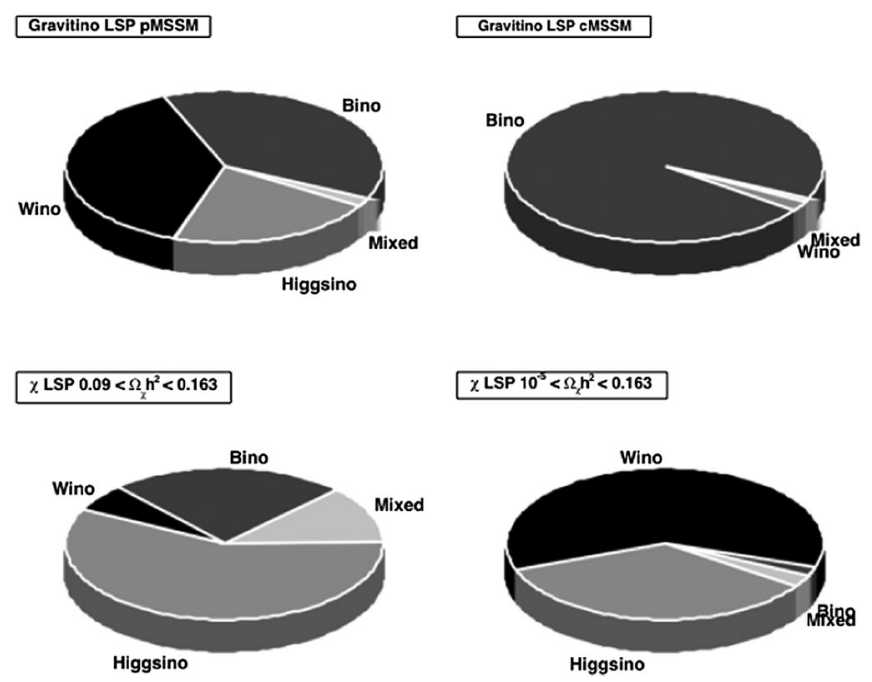

FIG. 5. Nature of the lightest neutralino for pMSSM (top left) and cMSSM (top right) points with gravitino LSP and pMSSM with neutralino LSP with tight (bottom left) and loose (bottom right) relic DM constraints. 
sample of points with the lightest neutralino being wino and Higgsino in $61 \%$ and $35 \%$ of the cases, respectively. This should be contrasted with the case of gravitino LSP models, characterized by an even distribution of points with different neutralino nature, from bino (38\%) to wino (39\%) and Higgsino (22\%) (see Fig. 5). In contrast to all these pMSSM scenarios, the gravitino model in the constrained MSSM is basically restricted to the case of bino-like neutralinos, which accounts for 97\% of the cMSSM accepted points, as shown in Fig. 5.

\section{RESULTS}

The viable regions of the MSSM parameter space with gravitino LSP excluded by the LHC run-1 searches and those projected for 300 and $3000 \mathrm{fb}^{-1}$ of data at $14 \mathrm{TeV}$, in the case no excess of events above the SM backgrounds will be observed, have been studied for various combinations of parameters. We present the results by computing the fractions of our scan points which are (will be) excluded by the LHC searches given in Table IV. Quantitatively, the values of these fractions depend on the range of parameters used in the scans. However, qualitatively the results hold when varying these ranges. The regions highlighted as being deeply probed by the LHC searches remain so even if the SUSY particle masses are pushed to higher values, although the fraction of points excluded will vary accordingly. A first observation derived from these results is the existence of a significant parameter space with neutralino NLSP when studying the pMSSM in sharp contrast with the results reported in previous studies using the cMSSM and fixed values of $\tan \beta$ and $m_{\tilde{G}}$. The sensitivity of the LHC data in falsifying the currently viable MSSM points with gravitino LSP can be contrasted with that for models with neutralino LSP. In the previous section, we have analyzed the difference in the nature of the lightest neutralino for the two models. Here, we concentrate on the fraction of points with SUSY masses up to $5 \mathrm{TeV}$ excluded by the LHC searches. Figure 6 and Table V summarize our findings, with the regions in grey being not accessible with the statistics of our scans. The distributions of the viable points from the scans and their fraction excluded by a negative result with $300 \mathrm{fb}^{-1}$ at $14 \mathrm{TeV}$ in the gravitino LSP and neutralino LSP scenarios with tight dark matter relic density constraint are shown by the figure in the plane of the gluino mass versus the neutralino mass. We see here that the reach in neutralino mass is similar in the two cases and reaches approximately $1 \mathrm{TeV}$, while in the direction of the gluino mass, the LHC will be able to test better the large mass regions in the case of a neutralino LSP and DM. We trace this back to the fact that in the case of a light neutralino DM, more supersymmetric particles have to be relatively light (e.g. the charged Higgsino/winos for the case of Higgsino/wino neutralino) and can give rise to detectable signals even if the colored states are very heavy.
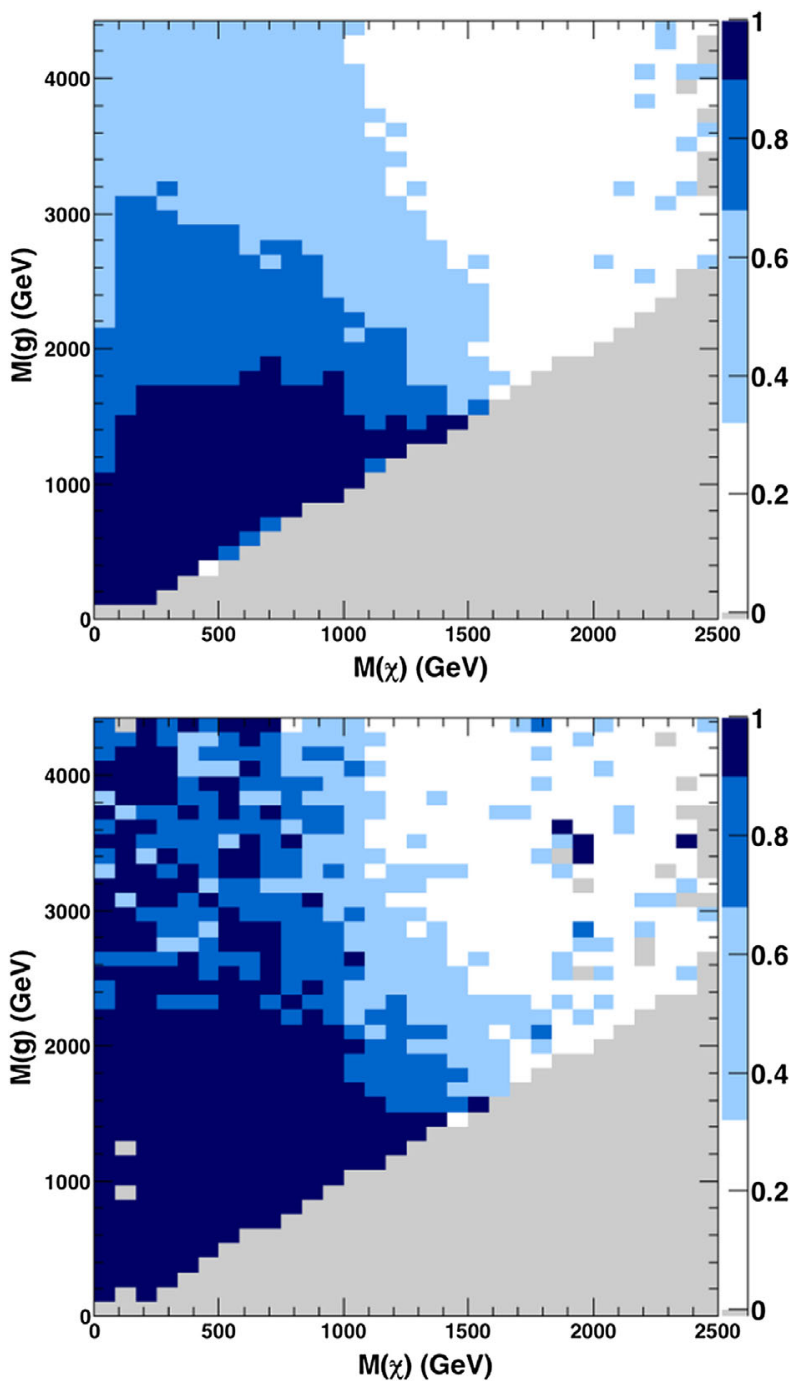

FIG. 6 (color online). Projected fractions of model points excluded by the LHC SUSY searches with $300 \mathrm{fb}^{-1}$ at $14 \mathrm{TeV}$ for the gravitino LSP (top) and the neutralino LSP scenario (bottom) in the plane defined by the gluino and lightest neutralino masses. For the neutralino LSP scenario only points consistent with the tight $\Omega_{\chi} h^{2}$ constraints and with neutralino scattering cross section $\sigma_{\chi p}^{S I}$ consistent with the LUX data are considered. The regions in grey have no points found in our scans.

The fractions of points excluded by the analyses at 8 , $14 \mathrm{TeV}$ LHC and HL-LHC are given in Table $\mathrm{V}$ for gravitino LSP and neutralino LSP with the tight and loose dark matter relic density constraints. Since the composition of the sample of accepted points has significant differences in the nature of the lightest neutralino, as discussed above, these fractions are also given restricting the analysis to the points which have bino-, wino- and Higgsino-like neutralinos. We observe that the fraction of excluded gravitino LSP points is significantly larger (by $\sim 22 \%$ for the run-1 data and almost $75 \%$ at $14 \mathrm{TeV}$ ) than that of models with neutralino LSP and tight relic dark matter constraints. This 
TABLE V. Fraction of pMSSM points with gravitino LSP and neutralino LSP fulfilling the flavor physics and low energy data constraints excluded by LHC searches.

\begin{tabular}{lccc}
\hline \hline & $\tilde{G}$ LSP & $\tilde{\chi}^{0}$ LSP & $\tilde{\chi}^{0}$ LSP \\
\hline 8 TeV 25 fb & & $09<\Omega_{\chi} h^{2}<0.163$ & $10^{-5}<\Omega_{\chi} h^{2}<0.163$ \\
All & & & \\
Bino & 0.218 & 0.100 & 0.188 \\
Wino & 0.273 & 0.268 & 0.312 \\
Higgsino & 0.144 & 0 & 0.145 \\
14 TeV 300 fb & 0.245 & 0 & 0.249 \\
All & & & \\
Bino & 0.745 & 0.533 & 0.694 \\
Wino & 0.835 & 0.851 & 0.864 \\
Higgsino & 0.614 & 0.035 & 0.615 \\
14 TeV 3 ab & 0.808 & 0.343 & 0.811 \\
All & & & \\
Bino & 0.845 & 0.745 & 0.806 \\
Wino & 0.917 & 0.956 & 0.927 \\
Higgsino & 0.733 & 0.212 & 0.736 \\
\hline \hline
\end{tabular}

is largely due to the effect of the Higgsino-like neutralino component in the neutralino LSP sample. The Higgsino/ wino relic density naturally reaches the $\mathrm{CMB}$ value for neutralino LSP masses in the range $1.5-2.5 \mathrm{TeV}$. This pushes the SUSY spectrum to masses of $\sim 2 \mathrm{TeV}$ or more, i.e. at the limit of the LHC sensitivity or beyond. Therefore, if the $\Omega_{\chi} h^{2}$ constraints are enforced, the LHC sensitivity to Higgsino- and wino-like neutralinos LSP scenarios collapses. Indeed no points are excluded in this case from run-1 data, as seen in Table $\mathrm{V}$, and also the future runs will not be able to cover the whole parameter space. Of course in the case of the loose DM constraint, the neutralino mass is not pushed to such high values and the LHC is more effective in testing the model, at a level similar to that observed for the gravitino LSP scenario. The LHC searches are also particularly sensitive to the photino points, as well as to the other bino-like solutions. The fractions of these points removed by the run- 1 data and the projections to 300 and $3000 \mathrm{fb}^{-1}$ of data at $14 \mathrm{TeV}$ are $37 \%, 92 \%$ and $98 \%$, respectively. In the case of the gravitino scenario in the cMSSM, the projection for $300 \mathrm{fb}^{-1}$ at $14 \mathrm{TeV}$ has $34 \%$ of the points excluded if no signal is observed, when restricting the analysis to points with SUSY particle masses below $5 \mathrm{TeV}$ to cover a parameter space comparable to that used for the pMSSM. The difference in the fraction of excluded points is related to the distribution of the SUSY masses after applying all our selection criteria.

The gravitino and neutralino LSP MSSM scenarios can be probed and differentiated by combining the LHC and DM direct detection searches. In fact, in the case of gravitino LSP no signal from WIMP scattering on nucleons is expected in the underground DM searches. On the contrary, if the neutralino LSP is the WIMP DM particle, a large fraction of the points to which ATLAS and CMS will become sensitive at the LHC forthcoming runs are also expected to give a signal at the LUX [6] or LZ DM experiments $[112,113]$. The distribution of the fraction of points excluded by LHC with $300 \mathrm{fb}^{-1}$ of $14 \mathrm{TeV}$ data in the neutralino scattering cross section vs neutralino mass plane is shown in Fig. 7 for the gravitino and neutralino LSP and DM scenarios. We see here that, in the gravitino DM scenario with an NLSP mass below $1 \mathrm{TeV}$, a large part of the parameter points tested at LHC will also be tested by LZ and in that case the presence of a positive signal at collider without a direct detection counterpart will be a strong indication that the neutralino is not the LSP. For the case of a neutralino LSP and DM instead, the direct detection experiments are able to test also regions outside of the LHC sensitivity and detect heavy neutralinos, well beyond $1 \mathrm{TeV}$. Therefore, we observe that only a small fraction of points in our scan are able to evade both the LHC and the direct detection constraints. In quantitative terms, we find that $64(62) \%$ of the gravitino LSP and 82 (80)\% of the neutralino LSP pMSSM points to which the LHC experiments will be sensitive after integrating $300 \mathrm{fb}^{-1}\left(3000 \mathrm{fb}^{-1}\right)$ of data at $14 \mathrm{TeV}$ could be also tested by the planned LZ direct detection experiment, when the loose $\Omega_{\chi} h^{2}$ constraints are applied. In this case, it may be appropriate to rescale the $\chi$ scattering cross section for the neutralino LSP case by the ratio of the neutralino relic density to the $\mathrm{CMB}$ value. This lowers the fraction of points given above to $42 \%$ and $39 \%$, for 300 and $3000 \mathrm{fb}^{-1}$ respectively, in line with the result that we obtain when enforcing the tight relic density constraints.

Turning to the sensitivity over specific regions of the parameter space, the first set of variables we analyze is the relation of the neutralino relic density, mass and lifetime (see Figs. 8 and 9). The sensitivity to the neutralino nature 

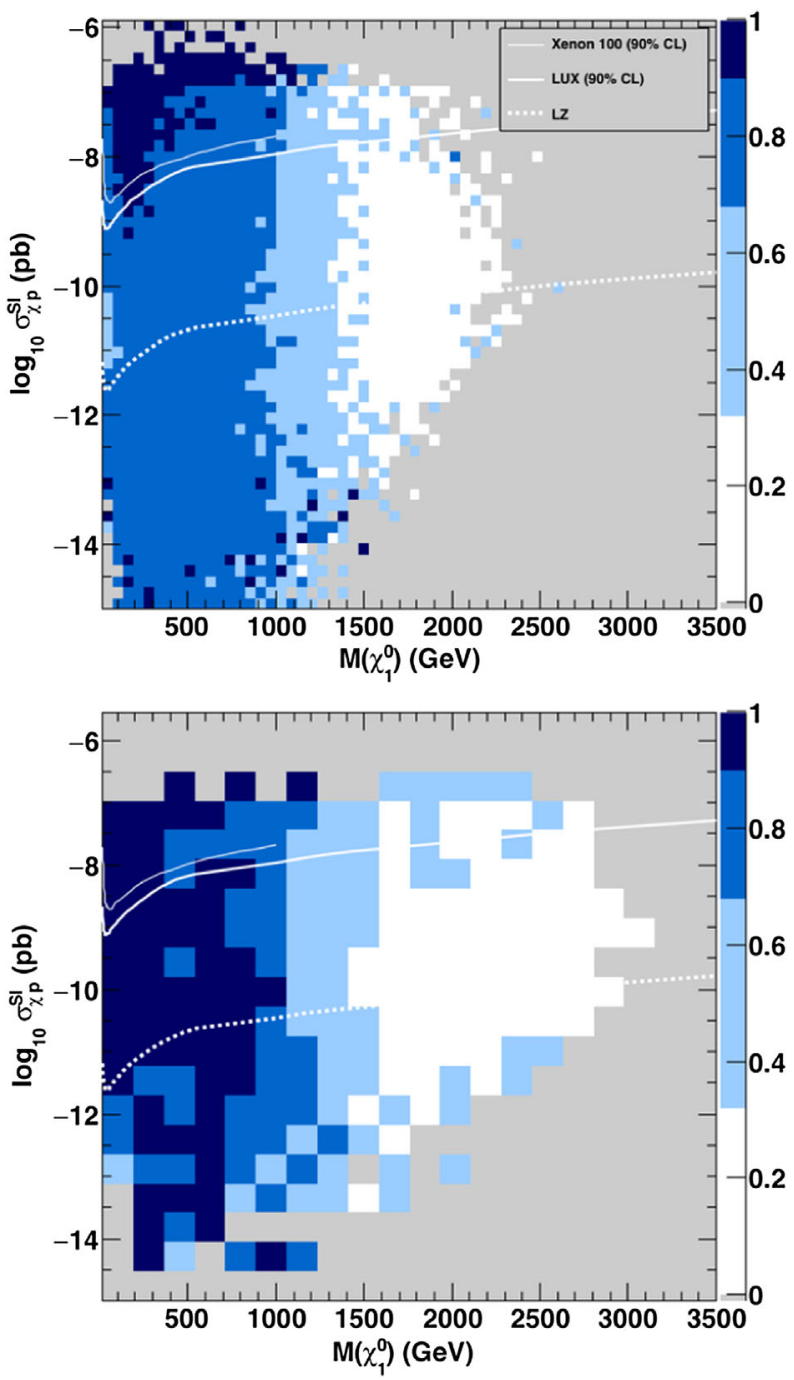

FIG. 7 (color online). Fractions of gravitino (top) and neutralino (bottom) LSP model points excluded by the LHC SUSY searches for the projection for $14 \mathrm{TeV}$ with $300 \mathrm{fb}^{-1}$ in the plane defined by the spin-independent neutralino scattering cross section and the neutralino mass. For the neutralino LSP the tight dark matter constraint is applied.

and mass is mapped, in part, onto these distributions. For example, the regions where the lightest neutralino is binolike are preferentially excluded resulting in the largest sensitivity from the LHC run-1 data and the projections for the $14 \mathrm{TeV}$ data being obtained for the parameter regions closest to the cosmological constraints in Fig. 8. Note on the other hand, comparing with Fig. 2, that also the region of wino NLSP will be well tested in the future runs. Moreover, the points with larger lifetime, just at the boundary of the constraint, correspond on average also to smaller neutralino mass and are more easily tested at a collider. Such a trend is clearly visible in Fig. 9.

In order to evaluate the sensitivity to the different assumptions used to derive the BBN constraints, we compare the results obtained using the constraints of
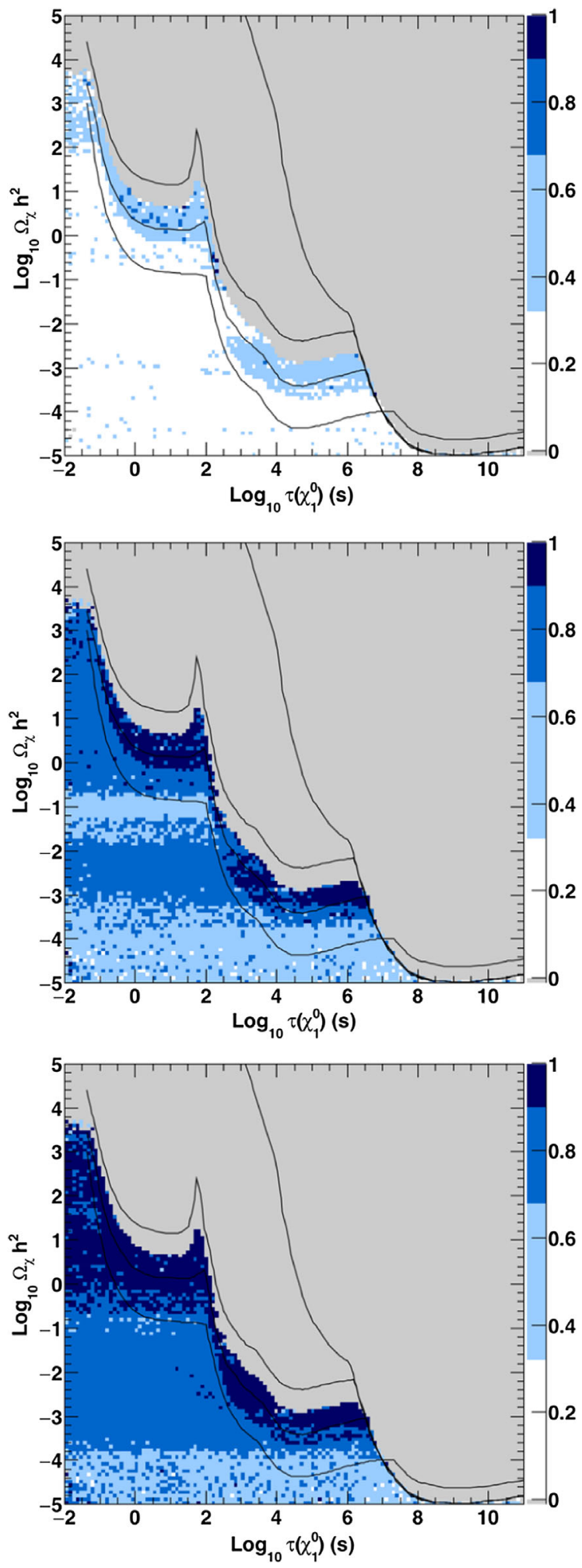

FIG. 8 (color online). Fractions of gravitino LSP model points excluded by the LHC SUSY searches with the $7+8 \mathrm{TeV}$ data (top) and the projection for $14 \mathrm{TeV}$ with $300 \mathrm{fb}^{-1}$ (center) and $3000 \mathrm{fb}^{-1}$ (bottom) in the plane defined by the neutralino relic density and the neutralino lifetime. The BBN constraints obtained following Ref. [74] are shown by the continuous lines corresponding to limits for neutralino hadronic branching fractions of $100 \%, 10 \%, 1 \%$ and $0 \%$ from bottom to top. 

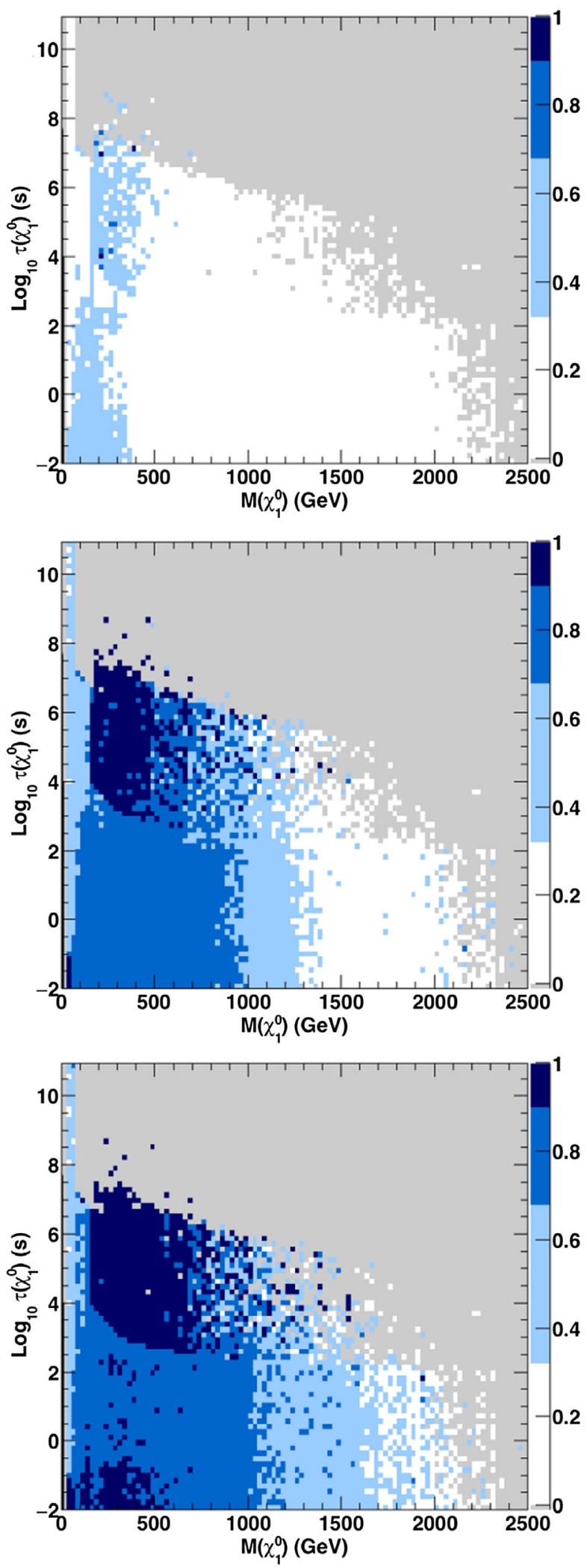

FIG. 9 (color online). Fractions of gravitino LSP model points excluded by the LHC SUSY searches with the $7+8 \mathrm{TeV}$ data (top) and the projection for $14 \mathrm{TeV}$ with $300 \mathrm{fb}^{-1}$ (center) and $3000 \mathrm{fb}^{-1}$ (bottom) in the plane defined by the neutralino mass and the neutralino lifetime.
Ref. [74], which are used throughout our study, to those of Ref. [19]. We find that the differences are minimal and restricted only to very particular points at the boundary. So the fraction of pMSSM points with gravitino LSP fulfilling the flavor physics and low energy data constraints excluded by LHC searches for these two implementations of the BBN bounds agree to better than 5\%. The two samples of points differ mostly in the particular value of the maximal gravitino mass, which does not affect the LHC phenomenology. Moreover, since we are not scanning systematically on that mass, but taking a comparable number of points at the boundary, such a difference does not influence substantially the fraction of excluded points.

The fractions of excluded points in the plane of the hadronic branching fraction as a function of the neutralino mass are shown in Fig. 10. Here, it is interesting to observe that the photino solutions can be largely excluded in the case the HL-LHC will not observe an excess of events in the SUSY searches: $97 \%$ of the points in our scan are excluded with $3000 \mathrm{fb}^{-1}$ at $14 \mathrm{TeV}$, as shown in the right panel of Fig. 10. But in general the sensitivity of the LHC in the gravitino DM scenario is again mostly determined by the neutralino NLSP mass scale rather than by its composition.

The LHC is also particularly sensitive to the test of gravitino LSP scenarios with relatively light NLSP neutralinos and large reheating temperature, $T_{\mathrm{RH}}$, close to the constraints derived from thermal leptogenesis, for which we assume the lower bounds of $2 \times 10^{9} \mathrm{GeV}[40,42]$ or $5 \times 10^{8} \mathrm{GeV}$, in the presence of flavor effects $[43,63]$, as shown in Fig. 11. Compared to cMSSM studies, which have essentially only bino $\chi$ NLSP as we have discussed above, the pMSSM scans generated for this analysis have points with wino $\chi$ LSP that enable one to increase the values of $T_{\mathrm{RH}}$ reachable in this scenario (see Fig. 3) and make the LHC sensitivity compelling for their test. These results are consistent with those obtained by the analysis of Ref. [75] that found a reheating temperature of order of $10^{9} \mathrm{GeV}$ for the case of $1.25 \mathrm{TeV}$ gluino and maximal gravitino mass around $70 \mathrm{GeV}$. In our scan we also reach larger gravitino masses, i.e. also larger $T_{\mathrm{RH}}$, since the neutralino relic density in the pMSSM reaches lower values than those obtained in [75].

Indeed a high reheating temperature is necessary to obtain the right gravitino abundance when the neutralino is light and has too low relic density to produce a sizable gravitino population in its decay. We see clearly in the figure also the complementary region of parameter space, where the whole gravitino density is indeed generated by the light neutralino decay and therefore the reheating temperature has to be very low. Both these two parameter regions will be completely tested in the next run of the LHC for neutralino masses below $500-700 \mathrm{GeV}$.

In these scenarios, the LHC sensitivity mostly comes from the constraints on the gluinos which have important 

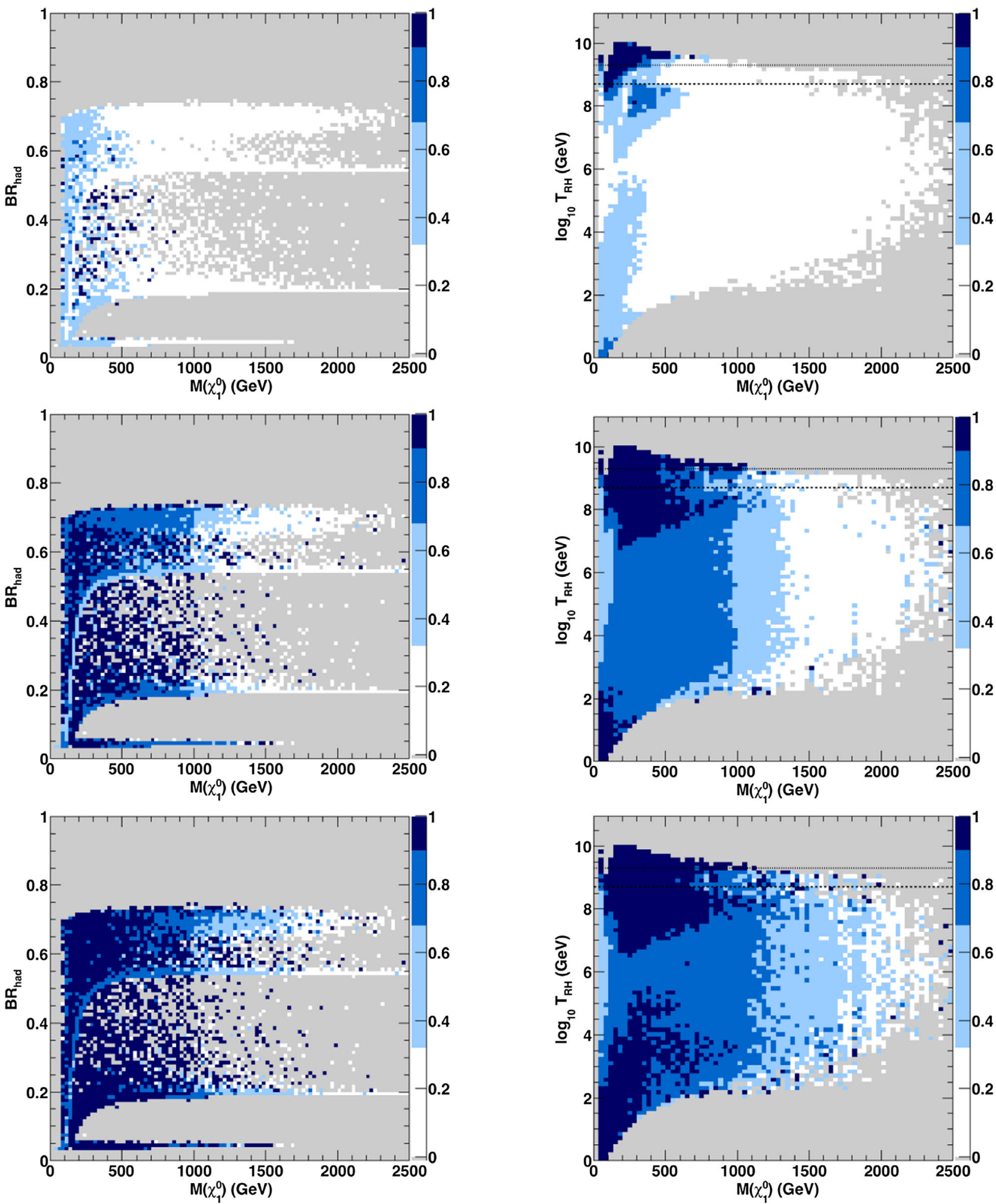

FIG. 10 (color online). Fractions of gravitino LSP points excluded by the LHC SUSY searches with the $7+8 \mathrm{TeV}$ data (top) and the projection for $14 \mathrm{TeV}$ with $300 \mathrm{fb}^{-1}$ (center) and $3000 \mathrm{fb}^{-1}$ (bottom) in the plane defined by the neutralino hadronic decay branching fraction and the neutralino mass. Note the photino solutions at small branching fraction values being almost completely excluded in the case of no SUSY signal at the

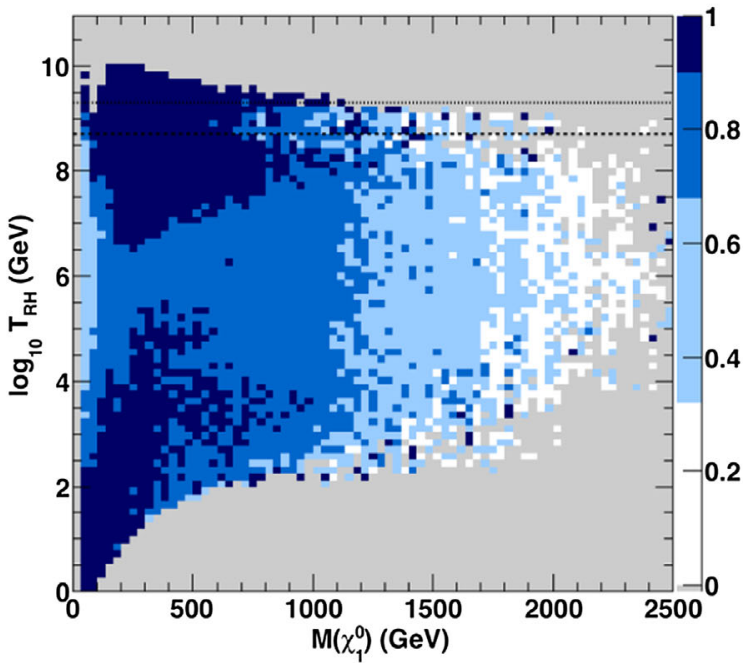

FIG. 11 (color online). Fractions of gravitino LSP points excluded by the LHC SUSY searches with the $7+8 \mathrm{TeV}$ data (top) and the projection for $14 \mathrm{TeV}$ with $300 \mathrm{fb}^{-1}$ (center) and $3000 \mathrm{fb}^{-1}$ (bottom) in the plane defined by the reheating temperature and the neutralino mass. The horizontal lines show the reheating temperature constraints from leptogenesis. 

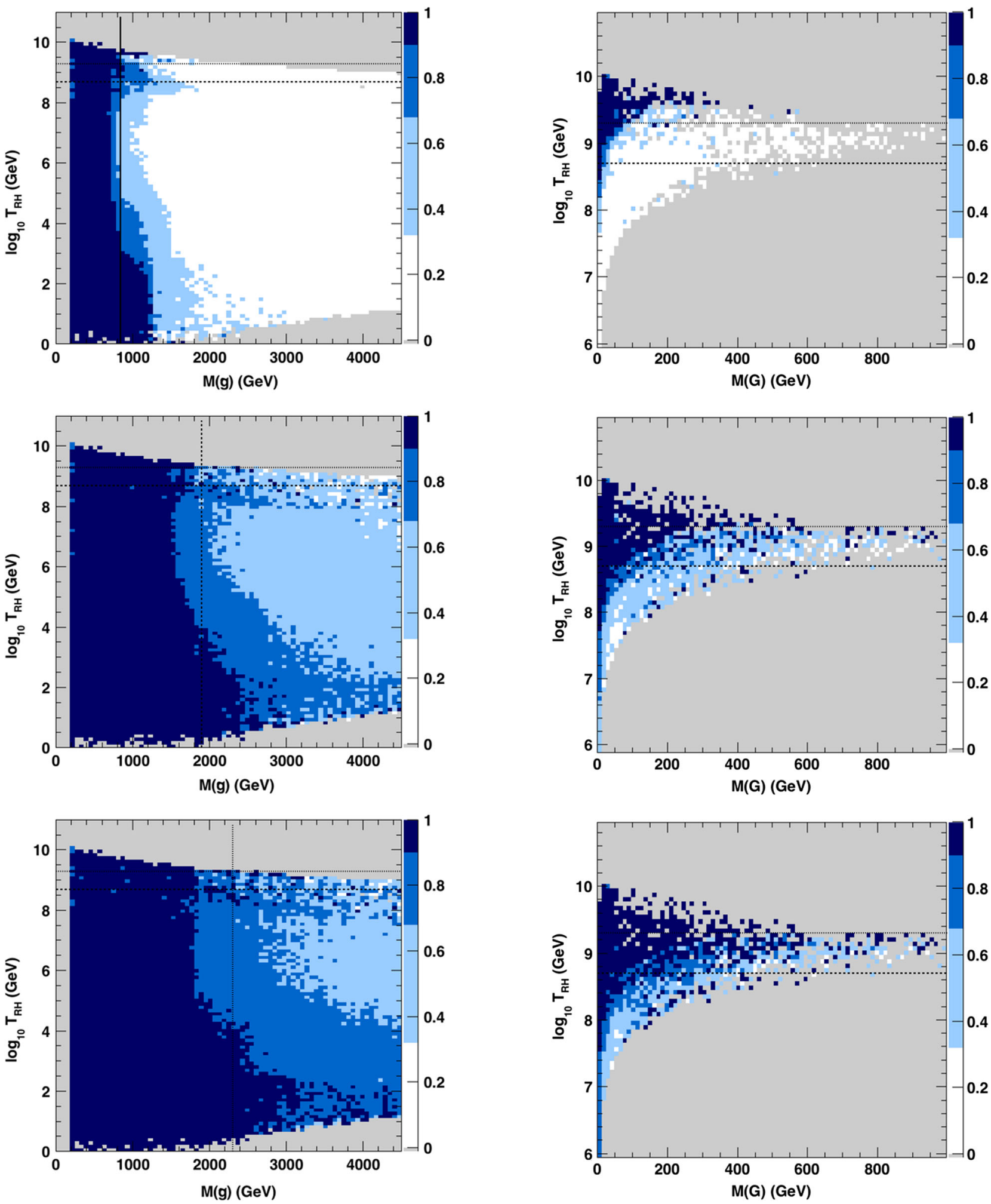

FIG. 12 (color online). Fractions of gravitino LSP points excluded by the LHC SUSY searches with the $7+8 \mathrm{TeV}$ data (top) and the projection for $14 \mathrm{TeV}$ with $300 \mathrm{fb}^{-1}$ (center) and $3000 \mathrm{fb}^{-1}$ (bottom) in the plane defined by the reheating temperature and the gluino mass. The horizontal lines show the reheating temperature constraints from leptogenesis and the vertical lines give the gluino mass value for which more than $95 \%$ of the points below it are excluded by the LHC SUSY searches.

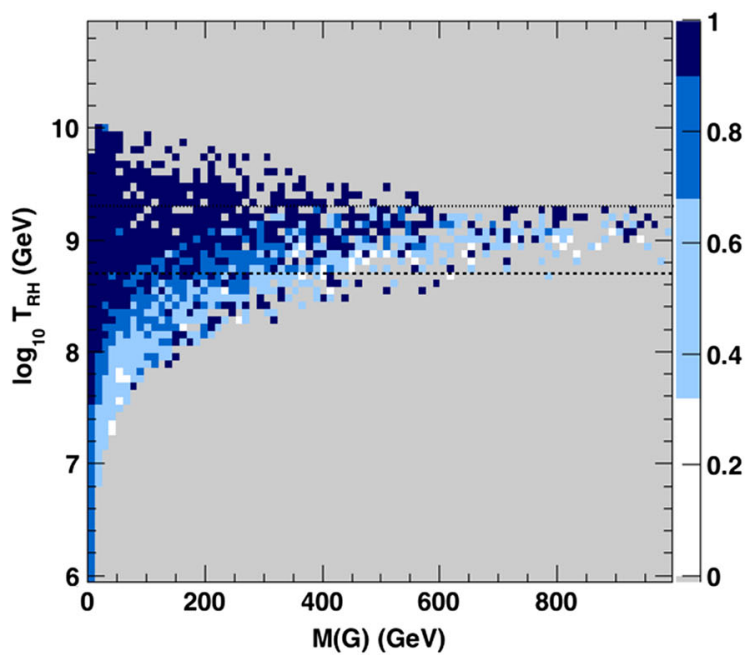

FIG. 13 (color online). Fractions of gravitino LSP points excluded by the LHC SUSY searches with the $7+8 \mathrm{TeV}$ data (top) and the projection for $14 \mathrm{TeV}$ with $300 \mathrm{fb}^{-1}$ (center) and $3000 \mathrm{fb}^{-1}$ (bottom) in the plane defined by the reheating temperature and the gravitino mass. The horizontal lines show the reheating temperature constraints from leptogenesis. 


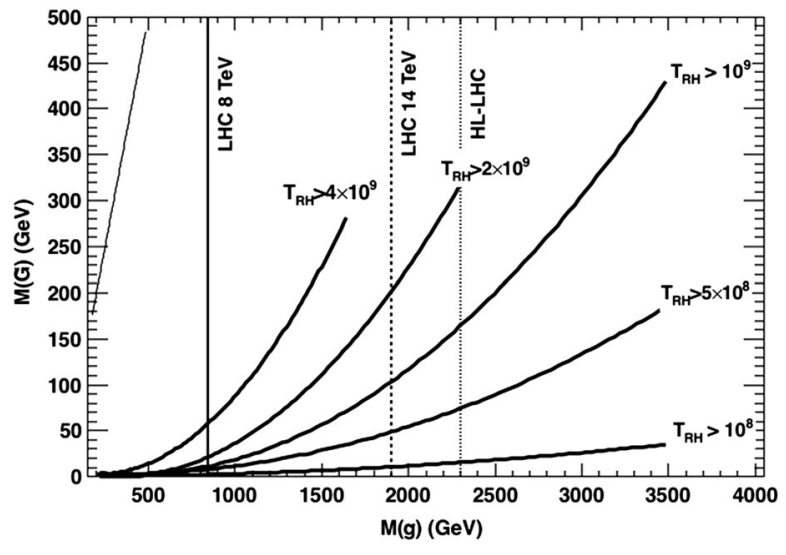

FIG. 14. Minimum values of the gravitino mass obtained in the gravitino LSP scans as a function of the gluino mass for different choices of the reheating temperature (in $\mathrm{GeV}$ ). The values of $2 \times 10^{9}$ and $5 \times 10^{8} \mathrm{GeV}$ correspond to the limits imposed by leptogenesis $[40,42,43,63]$. The thin diagonal line indicates the lower limit of the region where the gravitino is not the LSP. The vertical lines give the values of the gluino masses for which more than $95 \%$ of the scan points below them are excluded by the LHC SUSY searches at $7+8 \mathrm{TeV}$ (continuous) and projected at $14 \mathrm{TeV}$ for 300 (dashed) and $3000 \mathrm{fb}^{-1}$ (dotted), if no signal is observed.

implications on the viable values of the reheating temperature. The interplay of the gravitino and gluino masses and $T_{\mathrm{RH}}$ for the points of our scan after imposing the current and expected LHC constraints in the absence of a signal is highlighted in Figs. 12 and 13.

From Fig. 12, we can see clearly that the requirement of a specific reheating temperature and the LHC exclusion on the gluino mass are completely orthogonal constraints. In the upper $T_{\mathrm{RH}}$ region we point out that the boundary of the sample points is determined by the overclosure bound $\Omega_{\tilde{G}} h^{2}<0.163$ : since the gravitino density is increased both by large reheating temperature and gluino mass, as given in Eq. (1), such a constraint imposes an anticorrelation between the maximal temperature allowed and the gluino mass. Note that the fact that the low reheating temperature region is tested also at very large gluino masses, well beyond the LHC reach, is connected to the presence of a parameter region where the gravitinos are produced via neutralino decay and in that case neither the LHC constraints nor the gravitino density are derived from the colored sector of the model. In Fig. 13 we can see instead the points in our scan along the gravitino mass. Since we took mostly values of the gravitino mass around the BBN bounds, the distribution is not uniform in this direction. We note that here the points corresponding to low $T_{\mathrm{RH}}$ have mostly a very small gravitino mass. The particular behavior of the upper bound is not due, in this case, directly to the overclosure bound, which would give $T_{\mathrm{RH}}^{\max } \propto M_{\tilde{G}}$, but to the interplay of the overclosure bound with the BBN constraints.
As we have discussed, the contribution of heavy gluinos to the production of gravitinos after inflation affects the $\Omega_{\tilde{G}} h^{2}$ value, as can be seen from Eq. (1) and LHC bounds, if combined with the additional requirement of successful thermal leptogenesis may effectively test the resulting gravitino LSP scenarios [34,41]. This is highlighted in Fig. 14, which summarizes the results of our scans by giving the contours for the minimum values of the gravitino masses obtained as a function of the gluino mass for different choices of $T_{\mathrm{RH}}$. This plot can be read in two complementary ways. First, by requiring a particular minimal reheating temperature together with the relic density constraint, we can set strong bounds on the gluino and gravitino masses, since these determine the gravitino abundance in most of our parameter space. For gluinos heavier than $800 \mathrm{GeV}$, satisfying the present LHC exclusion bounds, the gravitino mass has also to be substantial for reheating temperature above $10^{9} \mathrm{GeV}$. Conversely, for each gravitino mass value, a choice of $T_{\mathrm{RH}}$ corresponds to an upper bound on the gluino mass to avoid overclosure. Then, by comparing these contours with the reheating temperature needed by thermal leptogenesis discussed above, we observe that the LHC data should either observe a gluino signal or exclude the gravitino DM scenarios with gravitino mass below $200-300 \mathrm{GeV}$ and classical thermal leptogenesis, after accumulating sufficient statistics. In this study the availability of the data set anticipated from the HL-LHC operation is of crucial importance, since the sensitivity afforded by $3 \mathrm{ab}^{-1}$ of data at $14 \mathrm{TeV}$ would cover the region compatible with the leptogenesis data up to at least $M_{\tilde{G}} \simeq 300 \mathrm{GeV}$ for a reheating temperature bound of $T_{\mathrm{RH}}>2 \times 10^{9} \mathrm{GeV}$ and more for higher values of $T_{\mathrm{RH}}$. In this case, the lack of observation of a gluino in the LHC data would basically exclude most of the gravitino LSP scenarios discussed here for the reheating temperatures required by thermal leptogenesis. Therefore, gravitino LSP models, in conjunction with constraints derived from leptogenesis, offer a strong motivation for the HL-LHC program. Scenarios of flavored leptogenesis, allowing for lower $T_{\mathrm{RH}} \geq 5 \times 10^{8} \mathrm{GeV}$, may still accommodate gluino masses even larger than those which can be probed at the HL-LHC, but could still be probed for gravitino masses below $\simeq 60 \mathrm{GeV}$.

\section{CONCLUSIONS}

The search for dark matter is tightly connected to that of new physics beyond the SM. These searches bring together the efforts and the data accumulated by the LHC experiments but also the DM underground direct searches. Gravitino dark matter represents a compelling scenario in supersymmetry. In this paper we have presented a detailed study of the gravitino LSP and dark matter scenario within the phenomenological MSSM and contrasted it with the solutions available in the CMSSM. Cosmological bounds on the scenario result in upper bounds on the 
gravitino mass and the reheating temperature necessary to fulfill the requirement of a DM energy density in agreement with CMB data.

Our analysis focused on the large gravitino mass region, at the boundary of the nucleosynthesis constraints, because that part of the parameter space allows for the largest possible values of the reheating temperature. The present and projected LHC constraints considered in this study include the monojet bounds that are important to test the parameter regions with large mass degeneracy, in particular the case of Higgsino and wino NLSP. The use of the unconstrained PMSSM model with $19+1$ free parameters has scenarios with bino-, winoand Higgsino-like NLSP, which are not available to studies carried out in the constrained MSSM with five parameters which yield almost uniquely solutions with bino-like NLSP neutralinos. We find that the scenario with gravitino LSP dark matter in the pMSSM is characterized by a very different composition of the neutralino NLSP in comparison to the case of a neutralino LSP. In particular all three types of compositions are nearly equivalently possible in the gravitino DM scenario, while for a WIMP neutralino a Higgsino composition is preferred. Such a specific composition is unfortunately more difficult to test at a collider, since the DM mass scale tends to be above $1 \mathrm{TeV}$. On the other hand, direct detection experiments could give a future detection, even for large neutralino mass, and cover a large part of the yet allowed parameter space. But if no signal appears in searches at those experiments and in direct production at the LHC, strong constraints on the neutralino WIMP scenario will be obtained, making the gravitino LSP scenarios particularly interesting. We have seen that in the case of gravitino DM and neutralino NLSP, the absence of a direct detection signal should allow one to exclude the neutralino DM scenario and point to an unstable NLSP in a large part of the parameter space.

The interplay of the LHC and cosmology data can be pursued even further in gravitino dark matter scenarios. The relation between the gravitino relic density and the gluino or gaugino masses provides us with an efficient way to test also the cosmological scenario. The region of the pMSSM parameter space with large gluino masses, beyond the projected LHC sensitivity, corresponds to gravitino energy densities that are too large at the high reheating temperatures required by thermal leptogenesis. Therefore, if the next runs of the LHC and especially the high-luminosity operation will not observe any signal of gluino direct production, the high reheating temperature region will be excluded for gravitino masses below $200 \mathrm{GeV}$.

\section{ACKNOWLEDGMENTS}

A. A., L. C. and F. M. acknowledge partial support from the European Union FP7 ITN INVISIBLES (Marie Curie Actions, PITN-GA-2011-289442), J. H. from the German Academy of Science through the Leopoldina Fellowship Programme under Grant No. LPDS 2012-14.
[1] G. Aad et al. (ATLAS Collaboration), Observation of a new particle in the search for the Standard Model Higgs boson with the ATLAS detector at the LHC, Phys. Lett. B 716, 1 (2012).

[2] S. Chatrchyan et al. (CMS Collaboration), Observation of a new boson at a mass of $125 \mathrm{GeV}$ with the CMS experiment at the LHC, Phys. Lett. B 716, 30 (2012).

[3] P. A. R. Ade et al. (Planck Collaboration), Planck 2013 results. XVI. Cosmological parameters, Astron. Astrophys. 571, A16 (2014).

[4] Z. Ahmed et al. (CDMS-II Collaboration), Dark matter search results from the CDMS II experiment, Science 327, 1619 (2010).

[5] E. Aprile et al. (XENON100 Collaboration), Dark Matter Results from 225 Live Days of XENON100 Data, Phys. Rev. Lett. 109, 181301 (2012).

[6] D. S. Akerib et al. (LUX Collaboration), First Results from the LUX Dark Matter Experiment at the Sanford Underground Research Facility, Phys. Rev. Lett. 112, 091303 (2014).

[7] P. Bechtle et al., Constrained supersymmetry after the Higgs boson discovery: A global analysis with Fittino, Proc. Sci., EPS-HEP2013 (2013) 313 [arXiv:1310.3045].
[8] O. Buchmueller et al., The CMSSM and NUHM1 after LHC run 1, Eur. Phys. J. C 74, 2922 (2014).

[9] L. Roszkowski, E. M. Sessolo, and A. J. Williams, What next for the CMSSM and the NUHM: Improved prospects for superpartner and dark matter detection, J. High Energy Phys. 08 (2014) 067.

[10] A. Djouadi et al. (MSSM Working Group Collaboration), The minimal supersymmetric standard model: Group summary report, arXiv:hep-ph/9901246.

[11] S. Henrot-Versille, R. Lafaye, T. Plehn, M. Rauch, D. Zerwas, S. Plaszczynski, B. Rouille d'Orfeuil, and M. Spinelli, Constraining supersymmetry using the relic density and the Higgs boson, Phys. Rev. D 89, 055017 (2014).

[12] N. Craig, The state of supersymmetry after run I of the LHC, arXiv:1309.0528.

[13] P. Nath, Supersymmetry after the Higgs, arXiv: 1501.01679.

[14] S. S. AbdusSalam, B. C. Allanach, F. Quevedo, F. Feroz, and M. Hobson, Fitting the phenomenological MSSM, Phys. Rev. D 81, 095012 (2010).

[15] J. A. Conley, J. S. Gainer, J. L. Hewett, M. P. Le, and T. G. Rizzo, Supersymmetry without prejudice at the $7 \mathrm{TeV}$ LHC, arXiv:1103.1697. 
[16] A. Arbey, M. Battaglia, and F. Mahmoudi, Implications of LHC searches on SUSY particle spectra: The pMSSM parameter space with neutralino dark matter, Eur. Phys. J. C 72, 1847 (2012).

[17] S. Sekmen, S. Kraml, J. Lykken, F. Moortgat, S. Padhi, L. Pape, M. Pierini, H. B. Prosper, and M. Spiropulu, Interpreting LHC SUSY searches in the phenomenological MSSM, J. High Energy Phys. 02 (2012) 075.

[18] A. Arbey, M. Battaglia, and F. Mahmoudi, Constraints on the MSSM from the Higgs sector: A pMSSM study of Higgs searches, $B_{s}^{0} \rightarrow \mu^{+} \mu^{-}$and dark matter direct detection, Eur. Phys. J. C 72, 1906 (2012).

[19] M. W. Cahill-Rowley, J. L. Hewett, S. Hoeche, A. Ismail, and T. G. Rizzo, The new look pMSSM with neutralino and gravitino LSPs, Eur. Phys. J. C 72, 2156 (2012).

[20] M. W. Cahill-Rowley, J. L. Hewett, A. Ismail, and T. G. Rizzo, The Higgs sector and fine-tuning in the pMSSM, Phys. Rev. D 86, 075015 (2012).

[21] A. Arbey, M. Battaglia, A. Djouadi, and F. Mahmoudi, The Higgs sector of the phenomenological MSSM in the light of the Higgs boson discovery, J. High Energy Phys. 09 (2012) 107.

[22] M. W. Cahill-Rowley, J. L. Hewett, A. Ismail, and T. G. Rizzo, More energy, more searches, but the phenomenological MSSM lives on, Phys. Rev. D 88, 035002 (2013).

[23] A. Arbey, M. Battaglia, A. Djouadi, and F. Mahmoudi, An update on the constraints on the phenomenological MSSM from the new LHC Higgs results, Phys. Lett. B 720, 153 (2013).

[24] A. Arbey, M. Battaglia, and F. Mahmoudi, Complementarity of WIMP sensitivity with direct SUSY, monojet and dark matter searches in the MSSM, Phys. Rev. D 89, 077701 (2014).

[25] K. Kowalska, L. Roszkowski, E. M. Sessolo, and S. Trojanowski, Low fine-tuning in the MSSM with Higgsino dark matter and unification constraints, J. High Energy Phys. 04 (2014) 166.

[26] J. Heisig, J. Kersten, B. Panes, and T. Robens, A survey for low stau yields in the MSSM, J. High Energy Phys. 04 (2014) 053.

[27] J. Heisig, Gravitino LSP and leptogenesis after the first LHC results, J. Cosmol. Astropart. Phys. 04 (2014) 023.

[28] CMS Collaboration, Phenomenological MSSM interpretation of the CMS 2011 5fb-1 results, Report No. CMSPAS-SUS-12-030.

[29] CMS Collaboration, Phenomenological MSSM interpretation of the CMS 7 and $8 \mathrm{TeV}$ results, Report No. CMSPAS-SUS-13-020.

[30] W. Buchmuller, L. Covi, K. Hamaguchi, A. Ibarra, and T. Yanagida, Gravitino dark matter in R-parity breaking vacua, J. High Energy Phys. 03 (2007) 037.

[31] M. Bolz, A. Brandenburg, and W. Buchmuller, Thermal production of gravitinos, Nucl. Phys. B606, 518 (2001); B790, 336(E) (2008).

[32] J. Pradler and F. D. Steffen, Thermal gravitino production and collider tests of leptogenesis, Phys. Rev. D 75, 023509 (2007).

[33] J. R. Ellis, K. A. Olive, Y. Santoso, and V. C. Spanos, Gravitino dark matter in the CMSSM, Phys. Lett. B 588, 7 (2004).
[34] L. Roszkowski, R. Ruiz de Austri, and K. Y. Choi, Gravitino dark matter in the CMSSM and implications for leptogenesis and the LHC, J. High Energy Phys. 08 (2005) 080.

[35] D. G. Cerdeno, K. Y. Choi, K. Jedamzik, L. Roszkowski, and R. Ruiz de Austri, Gravitino dark matter in the CMSSM with improved constraints from BBN, J. Cosmol. Astropart. Phys. 06 (2006) 005.

[36] R. H. Cyburt, J. R. Ellis, B. D. Fields, K. A. Olive, and V. C. Spanos, Bound-state effects on light-element abundances in gravitino dark matter scenarios, J. Cosmol. Astropart. Phys. 11 (2006) 014.

[37] K. Y. Choi, L. Roszkowski, and R. Ruiz de Austri, Determining reheating temperature at colliders with axino or gravitino dark matter, J. High Energy Phys. 04 (2008) 016.

[38] J. R. Ellis, K. A. Olive, and Y. Santoso, Sneutrino NLSP scenarios in the NUHM with gravitino dark matter, J. High Energy Phys. 10 (2008) 005.

[39] S. Bailly, K. Y. Choi, K. Jedamzik, and L. Roszkowski, A reanalysis of gravitino dark matter in the constrained MSSM, J. High Energy Phys. 05 (2009) 103.

[40] G. F. Giudice, A. Notari, M. Raidal, A. Riotto, and A. Strumia, Towards a complete theory of thermal leptogenesis in the SM and MSSM, Nucl. Phys. B685, 89 (2004).

[41] M. Fujii, M. Ibe, and T. Yanagida, Upper bound on gluino mass from thermal leptogenesis, Phys. Lett. B 579, 6 (2004).

[42] W. Buchmuller, P. Di Bari, and M. Plumacher, Leptogenesis for pedestrians, Ann. Phys. 315, 305 (2005).

[43] C. S. Fong, E. Nardi, and A. Riotto, Leptogenesis in the Universe, Adv. High Energy Phys. 2012, 1 (2012).

[44] J. Wess and J. Bagger, Supersymmetry and Supergravity (Princeton University Press, Princeton, NJ, 1992), ISBN: 978-0691025308.

[45] P. Binetruy, Supersymmetry: Theory, Experiment and Cosmology (Oxford University Press, New York, 2006), ISBN: 10: 0199652732.

[46] M. Viel, J. Lesgourgues, M. G. Haehnelt, S. Matarrese, and A. Riotto, Constraining warm dark matter candidates including sterile neutrinos and light gravitinos with WMAP and the Lyman-alpha forest, Phys. Rev. D 71, 063534 (2005).

[47] A. Boyarsky, J. Lesgourgues, O. Ruchayskiy, and M. Viel, Lyman-alpha constraints on warm and on warm-pluscold dark matter models, J. Cosmol. Astropart. Phys. 05 (2009) 012.

[48] J. L. Feng, M. Kamionkowski, and S. K. Lee, Light gravitinos at colliders and implications for cosmology, Phys. Rev. D 82, 015012 (2010).

[49] K. Markovic and M. Viel, Lyman- $\alpha$ forest and cosmic weak lensing in a warm dark matter universe, Pub. Astron. Soc. Aust. 31, e006 (2014).

[50] E. A. Baltz and H. Murayama, Gravitino warm dark matter with entropy production, J. High Energy Phys. 05 (2003) 067.

[51] S. Dimopoulos, M. Dine, S. Raby, and S. D. Thomas, Experimental Signatures of Low-Energy Gauge Mediated Supersymmetry Breaking, Phys. Rev. Lett. 76, 3494 (1996). 
[52] G. F. Giudice and R. Rattazzi, Theories with gauge mediated supersymmetry breaking, Phys. Rep. 322, 419 (1999).

[53] CMS Collaboration, Report No. CMS-PAS-SUS-12-018.

[54] ATLAS Collaboration, Report No. ATLAS-CONF-2014001.

[55] P. de Aquino, F. Maltoni, K. Mawatari, and B. Oexl, Light gravitino production in association with gluinos at the LHC, J. High Energy Phys. 10 (2012) 008.

[56] F. Maltoni, A. Martini, K. Mawatari, and B. Oexl, Signals of a superlight gravitino at the LHC, J. High Energy Phys. 04 (2015) 021.

[57] L. J. Hall, K. Jedamzik, J. March-Russell, and S. M. West, Freeze-in production of FIMP dark matter, J. High Energy Phys. 03 (2010) 080.

[58] C. Cheung, G. Elor, and L. Hall, Gravitino freeze-in, Phys. Rev. D 84, 115021 (2011).

[59] L. Covi, J. E. Kim, and L. Roszkowski, Axinos as Cold Dark Matter, Phys. Rev. Lett. 82, 4180 (1999).

[60] J. L. Feng, A. Rajaraman, and F. Takayama, Superweakly Interacting Massive Particles, Phys. Rev. Lett. 91, 011302 (2003).

[61] J. L. Feng, A. Rajaraman, and F. Takayama, SuperWIMP dark matter signals from the early universe, Phys. Rev. D 68, 063504 (2003).

[62] M. Fukugita and T. Yanagida, Baryogenesis without grand unification, Phys. Lett. B 174, 45 (1986).

[63] P. Di Bari, An introduction to leptogenesis and neutrino properties, Contemp. Phys. 53, 315 (2012).

[64] M. Flanz, E. A. Paschos, U. Sarkar, and J. Weiss, Baryogenesis through mixing of heavy Majorana neutrinos, Phys. Lett. B 389, 693 (1996).

[65] A. Pilaftsis, $C P$ violation and baryogenesis due to heavy Majorana neutrinos, Phys. Rev. D 56, 5431 (1997).

[66] M. Olechowski, S. Pokorski, K. Turzynski, and J. D. Wells, Reheating temperature and gauge mediation models of supersymmetry breaking, J. High Energy Phys. 12 (2009) 026.

[67] L. Covi, M. Olechowski, S. Pokorski, K. Turzynski, and J. D. Wells, Supersymmetric mass spectra for gravitino dark matter with a high reheating temperature, J. High Energy Phys. 01 (2011) 033.

[68] S. Weinberg, Cosmological Constraints on the Scale of Supersymmetry Breaking, Phys. Rev. Lett. 48, 1303 (1982).

[69] M. Y. Khlopov and A. D. Linde, Is it easy to save the gravitino?, Phys. Lett. 138B, 265 (1984).

[70] J. R. Ellis, D. V. Nanopoulos, and S. Sarkar, The cosmology of decaying gravitinos, Nucl. Phys. B259, 175 (1985).

[71] R. H. Cyburt, J. Ellis, B. D. Fields, F. Luo, K. A. Olive, and V.C. Spanos, Gravitino decays and the cosmological lithium problem in light of the LHC Higgs and supersymmetry searches, J. Cosmol. Astropart. Phys. 05 (2013) 014.

[72] K. Jedamzik and M. Pospelov, Big bang nucleosynthesis and particle dark matter, New J. Phys. 11, 105028 (2009).

[73] M. Kawasaki, K. Kohri, and T. Moroi, Big-bang nucleosynthesis and hadronic decay of long-lived massive particles, Phys. Rev. D 71, 083502 (2005).
[74] K. Jedamzik, Big bang nucleosynthesis constraints on hadronically and electromagnetically decaying relic neutral particles, Phys. Rev. D 74, 103509 (2006).

[75] L. Covi, J. Hasenkamp, S. Pokorski, and J. Roberts, Gravitino dark matter and general neutralino NLSP, J. High Energy Phys. 11 (2009) 003.

[76] A. Arbey and F. Mahmoudi, SuperIso relic: A program for calculating relic density and flavor physics observables in supersymmetry, Comput. Phys. Commun. 181, 1277 (2010).

[77] J. Hasenkamp and M. W. Winkler, NMSSM with gravitino dark matter to be tested at LHC, Nucl. Phys. B877, 419 (2013).

[78] L. Boubekeur, K. Y. Choi, R. Ruiz de Austri, and O. Vives, The degenerate gravitino scenario, J. Cosmol. Astropart. Phys. 04 (2010) 005.

[79] B.C. Allanach, SOFTSUSY: A program for calculating supersymmetric spectra, Comput. Phys. Commun. 143, 305 (2002).

[80] A. Djouadi, J. Kalinowski, and M. Spira, HDECAY: A program for Higgs boson decays in the standard model and its supersymmetric extension, Comput. Phys. Commun. 108, 56 (1998).

[81] M. Muhlleitner, A. Djouadi, and Y. Mambrini, SDECAY: A Fortran code for the decays of the supersymmetric particles in the MSSM, Comput. Phys. Commun. 168, 46 (2005).

[82] G. L. Kane, C. F. Kolda, L. Roszkowski, and J. D. Wells, Study of constrained minimal supersymmetry, Phys. Rev. D 49, 6173 (1994).

[83] J. R. Ellis, K. A. Olive, and Y. Santoso, Constraining supersymmetry, New J. Phys. 4, 32 (2002).

[84] F. Mahmoudi, SuperIso: A program for calculating the isospin asymmetry of $B \rightarrow K *$ gamma in the MSSM, Comput. Phys. Commun. 178, 745 (2008).

[85] F. Mahmoudi, SuperIso v2.3: A program for calculating flavor physics observables in supersymmetry, Comput. Phys. Commun. 180, 1579 (2009).

[86] G. Belanger, F. Boudjema, A. Pukhov, and A. Semenov, Dark matter direct detection rate in a generic model with micrOMEGAs 2.2, Comput. Phys. Commun. 180, 747 (2009).

[87] T. Sjostrand, S. Mrenna, and P.Z. Skands, A brief introduction to PYTHIA 8.1, Comput. Phys. Commun. 178, 852 (2008).

[88] J. Pumplin, D. R. Stump, J. Huston, H. L. Lai, P. M. Nadolsky, and W. K. Tung, New generation of parton distributions with uncertainties from global QCD analysis, J. High Energy Phys. 07 (2002) 012.

[89] J. Alwall, M. Herquet, F. Maltoni, O. Mattelaer, and T. Stelzer, MadGraph 5: Going beyond, J. High Energy Phys. 06 (2011) 128.

[90] J. de Favereau, C. Delaere, P. Demin, A. Giammanco, V. Lemaître, A. Mertens, and M. Selvaggi (DELPHES 3 Collaboration), DELPHES 3, A modular framework for fast simulation of a generic collider experiment, J. High Energy Phys. 02 (2014) 057.

[91] A. Arbey, M. Battaglia, F. Mahmoudi, and D. Martinez Santos, Supersymmetry confronts $B s \rightarrow m u+m u-$ : 
Present and future status, Phys. Rev. D 87, 035026 (2013).

[92] Y. Amhis et al. (Heavy Flavor Averaging Group Collaboration), Averages of B-Hadron, C-Hadron, and tau-lepton properties as of early 2012, arXiv:1207.1158.

[93] R. Aaij et al. (LHCb Collaboration), Measurement of the $B_{s}^{0} \rightarrow \mu^{+} \mu^{-}$Branching Fraction and Search for $B^{0} \rightarrow$ $\mu^{+} \mu^{-}$Decays at the LHCb Experiment, Phys. Rev. Lett. 111, 101805 (2013).

[94] S. Chatrchyan et al. (CMS Collaboration), Measurement of the $B(s) \rightarrow \mu^{+} \mu^{-}$Branching Fraction and Search for $B^{0} \rightarrow$ $\mu^{+} \mu^{-}$with the CMS Experiment, Phys. Rev. Lett. 111, 101804 (2013).

[95] LHCb and CMS Collaborations, Reports No. LHCbCONF-2013-012 and No. CMS-PAS-BPH-13-007.

[96] I. Adachi et al. (Belle Collaboration), Evidence for $B^{-} \rightarrow \tau^{-} \bar{\nu}_{\tau}$ with a Hadronic Tagging Method Using the Full Data Sample of Belle, Phys. Rev. Lett. 110, 131801 (2013).

[97] J. P. Lees et al. (BABAR Collaboration), Evidence of $B^{+} \rightarrow$ $\tau^{+} \nu$ decays with hadronic B tags, Phys. Rev. D 88, 031102 (2013).

[98] A. G. Akeroyd and F. Mahmoudi, Constraints on charged Higgs bosons from $D_{s}^{ \pm} \rightarrow \mu^{+} \mu^{-}$and $D_{s}^{ \pm} \rightarrow \tau^{+} \tau^{-}$, J. High Energy Phys. 04 (2009) 121.

[99] M. Antonelli et al. (FlaviaNet Working Group on Kaon Decays Collaboration), Precision tests of the Standard Model with leptonic and semileptonic kaon decays, Nucl. Phys. B, Proc. Suppl. 181-182, 83 (2008).
[100] H. N. Brown et al. (Muon g-2 Collaboration), Precise Measurement of the Positive Muon Anomalous Magnetic Moment, Phys. Rev. Lett. 86, 2227 (2001).

[101] A. L. Read, Presentation of search results: The CL(s) technique, J. Phys. G 28, 2693 (2002).

[102] ATLAS Collaboration, Report No. ATLAS-CONF-2013047.

[103] G. Aad et al. (ATLAS Collaboration), Search for direct top-squark pair production in final states with two leptons in pp collisions at $\sqrt{s}=8 \mathrm{TeV}$ with the ATLAS detector, J. High Energy Phys. 06 (2014) 124.

[104] ATLAS Collaboration, Report No. ATLAS-CONF-2013037.

[105] ATLAS Collaboration, Report No. ATLAS-CONF-2013053.

[106] ATLAS Collaboration, Report No. ATLAS-CONF-2013049.

[107] ATLAS Collaboration, Report No. ATLAS-CONF-2013035.

[108] ATLAS Collaboration, Report No. ATLAS-CONF-2013093.

[109] ATLAS Collaboration, Report No. ATLAS-CONF-2013068.

[110] CMS Collaboration, Report No. CMS PAS EXO-12-048.

[111] CMS Collaboration, Report No. CMS PAS HIG-13-021.

[112] D.C. Malling et al., After LUX: The LZ program, arXiv:1110.0103.

[113] P. Cushman et al., Working Group Report: WIMP dark matter direct detection, arXiv:1310.8327. 